\title{
Asymptotic behavior of the heavy quark form factors at higher order
}

\author{
J. Blümlein, ${ }^{1}$ P. Marquard, ${ }^{1}$ and N. Rana ${ }^{1,2}$ \\ ${ }^{1}$ Deutsches Elektronen-Synchrotron, DESY, Platanenallee 6, D-15738 Zeuthen, Germany \\ ${ }^{2}$ INFN, Sezione di Milano, Via Celoria 16, I-20133 Milano, Italy
}

(Received 21 October 2018; published 14 January 2019)

\begin{abstract}
In the asymptotic limit $Q^{2} \gg m^{2}$, the heavy quark form factors exhibit Sudakov behavior. We study the corresponding renormalization group equations of the heavy quark form factors which do not only govern the structure of infrared divergences but also control the high energy logarithms. This enables us to obtain the complete logarithmic three-loop and partial four-loop contributions to the heavy quark form factors in perturbative quantum chromodynamics.
\end{abstract}

DOI: 10.1103/PhysRevD.99.016013

\section{INTRODUCTION}

Amplitudes for hard scattering processes in quantum chromodynamics (QCD) at higher order do provide precise phenomenological predictions for scattering processes and also a clear insight into underlying principles such as factorization or the universality of infrared (IR) singularities. The latter properties can be used to resum large logarithmic contributions by applying the corresponding evolution equations either globally or in particular kinematic regions. Especially for massless scattering amplitudes, remarkable progress has been made in the understanding of the structure of IR divergences due to the interplay of the soft- and collinear dynamics. A first step was taken in [1], where a prediction for the singularities of two-loop amplitudes was given except the single pole in the dimensional variable $\varepsilon=(4-D) / 2$. Later, generalizations of this result for multiparton amplitudes was obtained in [2-4] beyond two-loop order. The IR structure is more prominent and interesting especially in the case of the form factors. The interplay of the soft and collinear anomalous dimensions building up the singular structure of the form factors was first noticed in [5] at two-loop order and was later established at three-loop order in [6]. In many following publications this was studied in detail; see e.g., [7] for references.

It is also of interest to generalize these considerations to the massive case. Here a first step was taken in [8] by obtaining the IR singularities for one-loop scattering amplitudes with massive partons. Next, in the asymptotic limit $Q^{2} \gg m^{2}$, a factorization theorem was proposed

Published by the American Physical Society under the terms of the Creative Commons Attribution 4.0 International license. Further distribution of this work must maintain attribution to the author(s) and the published article's title, journal citation, and DOI. Funded by SCOAP . in [9-11]. While a first step was taken in [12] to obtain the IR structure of a generic two-loop amplitude without considering the small mass limit, finally in [13] the general solution was presented for the singular structure of a generic scattering amplitude containing massless and massive partons.

The universality of these IR singularities, along with the factorization of QCD amplitudes, presents a rich structure even for the massive case. Especially in the asymptotic limit, the amplitudes with massive partons exhibit the Sudakov behavior. It was first studied in [10] and a general factorization formula was presented. Solving the renormalization group equation (RGE), Ref. [14] presented the asymptotic behavior of the heavy quark vector form factor in the leading color limit up to four-loop order.

In the present paper, we study the asymptotic behavior of the different heavy quark form factors following the method proposed for massless cases in $[15,16]$ and in the massive case of Ref. [10], maintaining the complete color structure to three-loop order and for leading color at four-loop order. From a phenomenological perspective, the closer understanding of heavy quark production plays a significant role in current studies in elementary particle physics, from precision measurements of the Standard Model parameters to the search for signals from beyond the Standard Model. Henceforth, there has been much attention during the last decade to obtain precise theoretical predictions for physical quantities involving heavy quarks; see also [17-24]. Important elements to all these predictions are the form factors. Dedicated work has been going on for decades to obtain the heavy quark form factors for different currents, namely vector, axial-vector, scalar, and pseudoscalar currents at two-loop order [25-30] and threeloop order [31-34] in perturbative QCD. Since the complete computations are very challenging, results obtained in certain kinematic limits form important checks to these calculations. 
We present the complete three-loop results for the form factors in the asymptotic limit, retaining all logarithmic orders. For the $\mathcal{O}\left(\varepsilon^{0} L^{0}\right)$ terms we obtain the leading color contributions. A partial result for the vector form factor has been given in [29] for three-loop order, where unknown coefficients $K_{I}$ at three-loop order were yet missing. We also present yet approximate four-loop results, combining all ingredients known at present and outline which missing terms still need to be calculated.

\section{THE SUDAKOV BEHAVIOR}

We consider the renormalized form factors $F_{I}$ arising in the decay of a colorless massive boson, $I$, of momentum $Q$ to a pair of heavy quarks of mass $m$ in the asymptotic limit $Q^{2} \gg m^{2}$. Here $Q^{2}$ is the center-of-mass energy squared and $I=V, A, S$, and $P$ indicates a vector, an axial-vector, a scalar, and a pseudoscalar boson, respectively. In the asymptotic limit the differences between the vector and axial-vector form factors and between scalar and pseudoscalar form factors vanish. Also the magnetic form factor in the vector and axial-vector case vanish in this limit. Henceforth, we therefore only consider the electric form factor $\left(F_{V}\right)$ for the vector current and the scalar form factor $\left(F_{S}\right)$,

$$
\begin{aligned}
& -i v_{Q} \delta^{c d} \gamma^{\mu} F_{V}^{c d}, \\
& -\frac{m}{v} s_{Q} \delta_{c d} F_{S}^{c d},
\end{aligned}
$$

where $v=\left(\sqrt{2} G_{F}\right)^{-1 / 2}$ is the vacuum expectation value, $v_{Q}$ and $s_{Q}$ are the heavy quark vector and scalar couplings, and $c, d$ are color indices. We consider $n_{l}$ light quarks and a single heavy quark and deal with the case of only massless QCD contributions to the heavy quark form factors, i.e., no internal massive lines.

In the asymptotic limit, the functions $\hat{F}_{I}\left(a_{s}(\mu), \frac{Q^{2}}{\mu^{2}}, \frac{m^{2}}{\mu^{2}}, \varepsilon\right)$ satisfy the following integro-differential equation [35]:

$$
\begin{aligned}
Q^{2} & \frac{\partial}{\partial Q^{2}} \ln \hat{F}_{I}\left(\frac{Q^{2}}{\mu^{2}}, \frac{m^{2}}{\mu^{2}}, a_{s}, \varepsilon\right) \\
& =\frac{1}{2}\left[K_{I}\left(\frac{m^{2}}{\mu^{2}}, a_{s}, \varepsilon\right)+G_{I}\left(\frac{Q^{2}}{\mu^{2}}, a_{s}, \varepsilon\right)\right] .
\end{aligned}
$$

Here $\hat{F}_{I}$ contains all logarithmic and infrared pole contributions of the respective form factor. The strong coupling constant $a_{s}$ in the $\overline{\mathrm{MS}}$ scheme obeys the scale evolution equation

$$
\frac{d a_{s}\left(\mu^{2}\right)}{d \ln \mu^{2}}=-\sum_{k=0}^{\infty} \beta_{k} a_{s}^{k+2}\left(\mu^{2}\right),
$$

where $\beta_{k}$ are the expansion coefficients of the QCD $\beta$-function [36-42], which depend on the Casimir operators $C_{A}=N_{C}, \quad C_{F}=\left(N_{C}^{2}-1\right) /\left(2 N_{C}\right) \quad$ and $\quad T_{F}=1 / 2 \quad$ in $S U\left(N_{C}\right)$ and the number of flavors $n_{l}$ up to three-loop order. The functions $K_{I}$ incorporate the contributions from the heavy quark mass $m$ and are process independent, whereas, the functions $G_{I}$ are process dependent. The functions $G_{I}$ and $K_{I}$ obey the renormalization group equations

$$
\begin{aligned}
\mu^{2} \frac{d}{d \mu^{2}} G_{I}\left(\frac{Q^{2}}{\mu^{2}}, a_{s}, \varepsilon\right) & =-\lim _{m \rightarrow 0} \mu^{2} \frac{d}{d \mu^{2}} K_{I}\left(\frac{m^{2}}{\mu^{2}}, a_{s}, \varepsilon\right) \\
& =A_{q}\left(a_{s}\left(\mu^{2}\right)\right)
\end{aligned}
$$

cf. also [10]. Individually one obtains

$$
\begin{aligned}
& K_{I}=K_{I}\left(a_{s}\left(m^{2}\right), 1, \varepsilon\right)-\int_{\frac{m^{2}}{\mu^{2}}}^{1} \frac{d \lambda}{\lambda} A_{q}\left(a_{s}\left(\lambda \mu^{2}\right)\right), \\
& G_{I}=G_{I}\left(a_{s}\left(Q^{2}\right), 1, \varepsilon\right)+\int_{\frac{Q^{2}}{\mu^{2}}} \frac{d \lambda}{\lambda} A_{q}\left(a_{s}\left(\lambda \mu^{2}\right)\right) .
\end{aligned}
$$

It is understood that all contributing functions obey series expansions of the kind

$$
\mathcal{A}_{I}\left(a_{s}\right)=\sum_{k=1}^{\infty} a_{s}^{k} \mathcal{A}_{I}^{(k)} .
$$

The coefficients $A_{q}^{(n)}$ are known up to three-loop order $[43,44]$. The leading color and complete $n_{l}^{2}, n_{l}^{3}$ contributions of $A_{q}^{(4)}$ were obtained in [45-50] recently. The finite functions $K_{I}, G_{I}$, and $A_{q}$ depend on $a_{s}\left(k^{2}\right)$ with the corresponding scales $k^{2}=m^{2}, Q^{2}, \lambda \mu^{2}$.

To solve Eq. (3), the following expansion is performed:

$\ln \hat{F}_{I}\left(a_{s}, \frac{Q^{2}}{\mu^{2}}, \frac{m^{2}}{\mu^{2}}, \varepsilon\right)=\sum_{n=1}^{\infty} a_{s}^{n}\left(\frac{m^{2}}{\mu^{2}}\right)^{-n \varepsilon} \mathcal{F}_{I}^{(n)}\left(\frac{Q^{2}}{m^{2}}, \frac{m^{2}}{\mu^{2}}, \varepsilon\right)$.

In the following we use $L \equiv \ln \left(Q^{2} / m^{2}\right)$ by setting $\mu^{2}=m^{2}$, for convenience; cf. also [14]. The $\mu^{2}$-dependence can be easily recovered. The nonlogarithmic contributions to the form factor are not contained in $\hat{F}_{I}$. They are obtained by matching

$F_{I}\left(a_{s}, \frac{Q^{2}}{\mu^{2}}, \frac{m^{2}}{\mu^{2}}, \varepsilon\right)=\mathcal{C}_{I}\left(a_{s}, \varepsilon\right) \hat{F}_{I}\left(a_{s}, \frac{Q^{2}}{\mu^{2}}, \frac{m^{2}}{\mu^{2}}, \varepsilon\right)$

to the complete form factors.

To have a more compact representation, the functions $\mathcal{F}_{I}^{(k)}$ in Eq. (8) are presented for the case of the unrenormalized coupling $\hat{a}_{s}$ here: 


$$
\begin{aligned}
& \mathcal{F}_{I}^{(1)}=\frac{1}{\varepsilon}\left[\left(-\frac{1}{2} K_{I}^{(1)}-\frac{1}{2} G_{I}^{(1)}\right)+\frac{1}{2} A_{I}^{(1)} L\right]+\frac{1}{2} G_{I}^{(1)} L-\frac{1}{4} A_{I}^{(1)} L^{2}+\varepsilon\left[-\frac{1}{4} G_{I}^{(1)} L^{2}+\frac{1}{12} A_{I}^{(1)} L^{3}\right] \\
&+\varepsilon^{2}\left[\frac{1}{12} G_{I}^{(1)} L^{3}-\frac{1}{48} A_{I}^{(1)} L^{4}\right]+\varepsilon^{3}\left[-\frac{1}{48} G_{I}^{(1)} L^{4}+\frac{1}{240} A_{I}^{(1)} L^{5}\right] . \\
& \mathcal{F}_{I}^{(2)}=\frac{1}{\varepsilon^{2}}\left[\left(-\frac{1}{4} K_{I}^{(1)} \beta_{0}-\frac{1}{4} G_{I}^{(1)} \beta_{0}\right)+\frac{1}{4} A_{I}^{(1)} \beta_{0} L\right]+\frac{1}{\varepsilon}\left[\left(-\frac{1}{4} K_{I}^{(2)}-\frac{1}{4} G_{I}^{(2)}\right)+\left(\frac{1}{4} A_{I}^{(2)}+\frac{1}{2} G_{I}^{(1)} \beta_{0}\right) L-\frac{1}{4} A_{I}^{(1)} \beta_{0} L^{2}\right] \\
&+\frac{1}{2} G_{I}^{(2)} L+\left(-\frac{1}{4} A_{I}^{(2)}-\frac{1}{2} G_{I}^{(1)} \beta_{0}\right) L^{2}+\frac{1}{6} A_{I}^{(1)} \beta_{0} L^{3}+\varepsilon\left[-\frac{1}{2} G_{I}^{(2)} L^{2}+\left(\frac{1}{6} A_{I}^{(2)}+\frac{1}{3} G_{I}^{(1)} \beta_{0}\right) L^{3}-\frac{1}{12} A_{I}^{(1)} \beta_{0} L^{4}\right] \\
&+\varepsilon^{2}\left[\frac{1}{3} G_{I}^{(2)} L^{3}+\left(-\frac{1}{12} A_{I}^{(2)}-\frac{1}{6} G_{I}^{(1)} \beta_{0}\right) L^{4}+\frac{1}{30} A_{I}^{(1)} \beta_{0} L^{5}\right] . \\
& \mathcal{F}_{I}^{(3)}=\frac{1}{\varepsilon^{3}}\left[\left(-\frac{1}{6} K_{I}^{(1)} \beta_{0}^{2}-\frac{1}{6} G_{I}^{(1)} \beta_{0}^{2}\right)+\frac{1}{6} A_{I}^{(1)} \beta_{0}^{2} L\right]+\frac{1}{\varepsilon^{2}}\left[\left(-\frac{1}{3} K_{I}^{(2)} \beta_{0}-\frac{1}{12} K_{I}^{(1)} \beta_{1}-\frac{1}{3} G_{I}^{(2)} \beta_{0}-\frac{1}{12} G_{I}^{(1)} \beta_{1}\right)\right. \\
&+\left.\left(\frac{1}{3} A_{I}^{(2)} \beta_{0}+\frac{1}{12} A_{I}^{(1)} \beta_{1}+\frac{1}{2} G_{I}^{(1)} \beta_{0}^{2}\right) L-\frac{1}{4} A_{I}^{(1)} \beta_{0}^{2} L^{2}\right]+\frac{1}{\varepsilon}\left[-\frac{1}{6}\left(K_{I}^{(3)}+G_{I}^{(3)}\right)\right. \\
&+\left.\left(\frac{1}{6} A_{I}^{(3)}+G_{I}^{(2)} \beta_{0}+\frac{1}{4} G_{I}^{(1)} \beta_{1}\right) L+\left(-\frac{1}{2} A_{I}^{(2)} \beta_{0}-\frac{1}{8} A_{I}^{(1)} \beta_{1}-\frac{3}{4} G_{I}^{(1)} \beta_{0}^{2}\right) L^{2}+\frac{1}{4} A_{I}^{(1)} \beta_{0}^{2} L^{3}\right] \\
&+ \frac{1}{2} G_{I}^{(3)} L+\left(-\frac{1}{4} A_{I}^{(3)}-\frac{3}{2} G_{I}^{(2)} \beta_{0}-\frac{3}{8} G_{I}^{(1)} \beta_{1}\right) L^{2}+\left(\frac{1}{2} A_{I}^{(2)} \beta_{0}+\frac{1}{8} A_{I}^{(1)} \beta_{1}+\frac{3}{4} G_{I}^{(1)} \beta_{0}^{2}\right) L^{3}-\frac{3}{16} A_{I}^{(1)} \beta_{0}^{2} L^{4} \\
&+ {\left[-\frac{3}{4} G_{I}^{(3)} L^{2}+\left(\frac{1}{4} A_{I}^{(3)}+\frac{3}{2} G_{I}^{(2)} \beta_{0}+\frac{3}{8} G_{I}^{(1)} \beta_{1}\right) L^{3}+\left(-\frac{3}{8} A_{I}^{(2)} \beta_{0}-\frac{3}{32} A_{I}^{(1)} \beta_{1}-\frac{9}{16} G_{I}^{(1)} \beta_{0}^{2}\right) L^{4}+\frac{9}{80} A_{I}^{(1)} \beta_{0}^{2} L^{5}\right] . }
\end{aligned}
$$

$$
\begin{aligned}
\mathcal{F}_{I}^{(4)}= & \frac{1}{\varepsilon^{4}}\left[\left(-\frac{1}{8} K_{I}^{(1)} \beta_{0}^{3}-\frac{1}{8} G_{I}^{(1)} \beta_{0}^{3}\right)+\frac{1}{8} A_{I}^{(1)} \beta_{0}^{3} L\right]+\frac{1}{\varepsilon^{3}}\left[\left(-\frac{3}{8} K_{I}^{(2)} \beta_{0}^{2}-\frac{1}{6} K_{I}^{(1)} \beta_{0} \beta_{1}-\frac{3}{8} G_{I}^{(2)} \beta_{0}^{2}-\frac{1}{6} G_{I}^{(1)} \beta_{0} \beta_{1}\right)\right. \\
& \left.+\left(\frac{3}{8} A_{I}^{(2)} \beta_{0}^{2}+\frac{1}{6} A_{I}^{(1)} \beta_{0} \beta_{1}+\frac{1}{2} G_{I}^{(1)} \beta_{0}^{3}\right) L-\frac{1}{4} A_{I}^{(1)} \beta_{0}^{3} L^{2}\right]+\frac{1}{\varepsilon^{2}}\left[\left(-\frac{3}{8} K_{I}^{(3)} \beta_{0}-\frac{1}{8} K_{I}^{(2)} \beta_{1}-\frac{1}{24} K_{I}^{(1)} \beta_{2}-\frac{3}{8} G_{I}^{(3)} \beta_{0}\right.\right. \\
& \left.-\frac{1}{8} G_{I}^{(2)} \beta_{1}-\frac{1}{24} G_{I}^{(1)} \beta_{2}\right)+\left(\frac{3}{8} A_{I}^{(3)} \beta_{0}+\frac{1}{8} A_{I}^{(2)} \beta_{1}+\frac{1}{24} A_{I}^{(1)} \beta_{2}+\frac{3}{2} G_{I}^{(2)} \beta_{0}^{2}+\frac{2}{3} G_{I}^{(1)} \beta_{0} \beta_{1}\right) L \\
& \left.+\left(-\frac{3}{4} A_{I}^{(2)} \beta_{0}^{2}-\frac{1}{3} A_{I}^{(1)} \beta_{0} \beta_{1}-G_{I}^{(1)} \beta_{0}^{3}\right) L^{2}+\frac{1}{3} A_{I}^{(1)} \beta_{0}^{3} L^{3}\right]+\frac{1}{\varepsilon}\left[\left(-\frac{1}{8} K_{I}^{(4)}-\frac{1}{8} G_{I}^{(4)}\right)\right. \\
& +\left(\frac{1}{8} A_{I}^{(4)}+\frac{3}{2} G_{I}^{(3)} \beta_{0}+\frac{1}{2} G_{I}^{(2)} \beta_{1}+\frac{1}{6} G_{I}^{(1)} \beta_{2}\right) L+\left(-\frac{3}{4} A_{I}^{(3)} \beta_{0}-\frac{1}{4} A_{I}^{(2)} \beta_{1}-\frac{1}{12} A_{I}^{(1)} \beta_{2}-3 G_{I}^{(2)} \beta_{0}^{2}-\frac{4}{3} G_{I}^{(1)} \beta_{0} \beta_{1}\right) L^{2} \\
& \left.+\left(A_{I}^{(2)} \beta_{0}^{2}+\frac{4}{9} A_{I}^{(1)} \beta_{0} \beta_{1}+\frac{4}{3} G_{I}^{(1)} \beta_{0}^{3}\right) L^{3}-\frac{1}{3} A_{I}^{(1)} \beta_{0}^{3} L^{4}\right]+\frac{1}{2} G_{I}^{(4)} L+\left(-\frac{1}{4} A_{I}^{(4)}-3 G_{I}^{(3)} \beta_{0}-G_{I}^{(2)} \beta_{1}-\frac{1}{3} G_{I}^{(1)} \beta_{2}\right) L^{2} \\
& +\left(A_{I}^{(3)} \beta_{0}+\frac{1}{3} A_{I}^{(2)} \beta_{1}+\frac{1}{9} A_{I}^{(1)} \beta_{2}+4 G_{I}^{(2)} \beta_{0}^{2}+\frac{16}{9} G_{I}^{(1)} \beta_{0} \beta_{1}\right) L^{3}+\left(-A_{I}^{(2)} \beta_{0}^{2}-\frac{4}{9} A_{I}^{(1)} \beta_{0} \beta_{1}-\frac{4}{3} G_{I}^{(1)} \beta_{0}^{3}\right) L^{4}+\frac{4}{15} A_{I}^{(1)} \beta_{0}^{3} L^{5} .
\end{aligned}
$$

The corresponding expression for the running coupling $a_{s}$ is obtained by replacing

$$
\hat{a}_{s}=1-a_{s} \frac{\beta_{0}}{\varepsilon}+a_{s}^{2}\left(\frac{\beta_{0}^{2}}{\varepsilon^{2}}-\frac{\beta_{1}}{2 \varepsilon}\right)+a_{s}^{3}\left(-\frac{\beta_{0}^{3}}{\varepsilon^{3}}+\frac{7}{6 \varepsilon^{2}} \beta_{0} \beta_{1}-\frac{\beta_{2}}{3 \varepsilon}\right)+\mathcal{O}\left(a_{s}^{4}\right)
$$

in Eqs. (8) and (9).

In massless scenarios, the soft $\left(f_{i}\right)$ and collinear $\left(B_{i}\right)$ anomalous dimensions of massless QCD govern the infrared structures, where $i \equiv q, g$ label quarks and gluons, respectively. In the case of two-parton amplitudes, e.g., the massless form 
factors, $\gamma_{i}=B_{i}+\frac{f_{i}}{2}$ is obtained from the leading pole $1 / \varepsilon$ and terms from lower orders. It is imperative that for the case of heavy quarks $\gamma_{q}$, along with similar contributions from the heavy quark anomalous dimension, will form the leading singularity. On the other hand, following soft-collinear effective theory, it was shown in [13] that the two-loop anomalous dimension matrix for two heavy parton correlations, which controls the singular structure, contains a heavy quark anomalous dimension $\gamma_{Q}$. Hence it is suggestive to form the following structure for the finite function:

$$
K_{I}^{(n)}=-2\left(\gamma_{q}^{(n)}+\gamma_{Q}^{(n)}-\gamma_{I}^{(n-1)}\right),
$$

where $\gamma_{q}=B_{q}+\frac{f_{q}}{2}$ and $B_{q}$ and $f_{q}$ are the collinear and soft anomalous dimension for massless quarks. They are known up to three-loop order $[5,43,44]$. Recently $\gamma_{q}^{(4)}$ in the color-planar limit was calculated in $[45,47,48]$ from the contributions to the four-loop massless form factor in addition to the $n_{l}^{2}$ and $n_{l}^{3}$ contributions. The anomalous dimension $\gamma_{I}^{(n-1)}$ is arising due to renormalization of the current. It vanishes for conserved currents, as the vector current. For the scalar current, the Yukawa coupling introduces the mass renormalization and hence $\gamma_{S}^{(n-1)}=\gamma_{m}^{(n-1)}$, being known up to four-loop order [51-57]. Furthermore, the renormalization constants $Z_{m, \mathrm{OS}}$ and $Z_{2, \mathrm{OS}}$ are given in [51,53,55,58,59], and [58-60], respectively.

The heavy quark anomalous dimension $\gamma_{Q}$ is connected with the soft Wilson-line operator and was known up to two-loop $[13,61,62]$. Also, it constitutes the non-logarithmic contribution of the massive cusp anomalous dimension in the asymptotic limit. Thus, we can obtain $\gamma_{Q}$ up to three-loop order from the known three-loop result [63]

$$
\begin{aligned}
\gamma_{Q}^{(0)}= & -2 C_{F} \\
\gamma_{Q}^{(1)}= & C_{F} C_{A}\left(-\frac{98}{9}+4 \zeta_{2}-4 \zeta_{3}\right)+C_{F} T_{F} n_{l}\left(\frac{40}{9}\right), \\
\gamma_{Q}^{(2)}= & C_{F} C_{A}^{2}\left(-\frac{343}{9}+\frac{608}{9} \zeta_{2}-\frac{88}{5} \zeta_{2}^{2}-\frac{740}{9} \zeta_{3}-8 \zeta_{2} \zeta_{3}+36 \zeta_{5}\right)+C_{F} C_{A} T_{F} n_{l}\left(\frac{356}{27}-\frac{160}{9} \zeta_{2}+\frac{496}{9} \zeta_{3}\right) \\
& +C_{F}^{2} T_{F} n_{l}\left(\frac{110}{3}-32 \zeta_{3}\right)+C_{F} T_{F}^{2} n_{l}^{2}\left(\frac{32}{27}\right) .
\end{aligned}
$$

Here $\zeta_{k}, k \geq 2, k \in \mathbb{N}$ are the values of the Riemann $\zeta$-function at integer argument. Note that, as mentioned in [13], the nonAbelian exponentiation theorem constrains the color structures of $\gamma_{Q}$. Hence, up to $\mathcal{O}\left(\alpha_{s}^{3}\right)$, the corresponding anomalous dimension for a massive color-octet boson $(G)$ is given by $\gamma_{G}=\frac{C_{A}}{C_{F}} \gamma_{Q}$. The other finite function $G_{I}^{(n)}$ only contains the information about the process and depends on $Q^{2}$ and not the heavy quark mass $m$. It is similar to the massless case of the Drell-Yan form factor

$$
G_{I}^{(n)}=2\left(B_{q}^{(n)}-\gamma_{I}^{(n-1)}\right)+f_{q}^{(n)}+C_{I}^{(n)}+\sum_{k=1}^{\infty} \varepsilon^{k} g_{I}^{n, k}
$$

Up to four-loop order, $C_{I}^{(n)}$ are given by

$$
\begin{aligned}
& C_{I}^{(1)}=0, \\
& C_{I}^{(2)}=\beta_{0} g_{I}^{1,1}, \\
& C_{I}^{(3)}=\beta_{1} g_{I}^{1,1}+\beta_{0}\left(g_{I}^{2,1}-\beta_{0} g_{I}^{1,2}\right), \\
& C_{I}^{(4)}=\beta_{2} g_{I}^{1,1}+\beta_{1}\left(g_{I}^{2,1}-2 \beta_{0} g_{I}^{1,2}\right)+\beta_{0}\left(g_{I}^{3,1}-\beta_{0} g_{I}^{2,2}+\beta_{0}^{2} g_{I}^{1,3}\right) .
\end{aligned}
$$

The components $g_{I}^{n, k}$ have to be extracted from the explicit result in this limit. Given the structural similarities, the functions $g_{I}^{n, k}$ are the same in both the massive and the massless case.

\section{PHENOMENOLOGICAL RESULTS}

Equations (12) and (13), along with (8) and (9), provide the three- and four-loop heavy quark form factors in the asymptotic limit. To obtain the vector and scalar form factors, we need $\mathcal{C}_{I}^{(3)}$ and $\mathcal{C}_{I}^{(4)}$, along with all the anomalous 
dimensions up to three and four loops and $g_{I}^{1, k}, g_{I}^{2, k}, g_{I}^{3, k}, \mathcal{C}_{I}^{1, k}$, and $\mathcal{C}_{I}^{2, k}$ to sufficient order $k$ in $\varepsilon$. As noted earlier, the coefficients $g_{I}^{n, k}$ can be obtained from the massless results; cf. [64,65]. The $\varepsilon^{0}$ part of $G_{I}^{(4)}$, as noted in Eq. (17), can also be obtained from the massless quark form factor. Below, we present $G_{V}^{(4)}$ and $G_{S}^{(4)}$. Note that $G_{S}^{(4)}$ constitutes the single pole in $\varepsilon$ of the Higgs-quark massless form factor.

$$
\begin{aligned}
G_{V}^{4}= & N_{C}^{4}\left(\frac{1913092765}{139968}+\frac{571537}{243} \zeta_{2}-\frac{2308}{5} \zeta_{2}^{2}+\frac{12350}{63} \zeta_{2}^{3}-\frac{169547}{18} \zeta_{3}+\frac{1622}{3} \zeta_{3}^{2}+\frac{8480}{9} \zeta_{3} \zeta_{2}-\frac{656}{5} \zeta_{3} \zeta_{2}^{2}\right. \\
& \left.+\frac{10100}{3} \zeta_{5}-288 \zeta_{5} \zeta_{2}-1410 \zeta_{7}\right)+N_{C}^{3} n_{l} T_{F}\left(-\frac{164021633}{11664}-\frac{1903985}{486} \zeta_{2}+\frac{7547}{15} \zeta_{2}^{2}-\frac{29432}{315} \zeta_{2}^{3}+\frac{406621}{81} \zeta_{3}\right. \\
& \left.+\frac{32}{3} \zeta_{3}^{2}-\frac{2432}{9} \zeta_{3} \zeta_{2}+\frac{1340}{9} \zeta_{5}\right)+C_{F}^{2} n_{l}^{2} T_{F}^{2}\left(\frac{693446}{243}-\frac{3232}{3} \zeta_{2}-\frac{21376}{45} \zeta_{2}^{2}+\frac{65632}{27} \zeta_{3}-\frac{512}{3} \zeta_{3} \zeta_{2}-\frac{832}{3} \zeta_{5}\right) \\
& +C_{F} C_{A} n_{l}^{2} T_{F}^{2}\left(\frac{8996449}{1458}+\frac{919544}{243} \zeta_{2}+\frac{2464}{15} \zeta_{2}^{2}-\frac{172832}{81} \zeta_{3}+\frac{1216}{9} \zeta_{3} \zeta_{2}-\frac{4928}{9} \zeta_{5}\right) \\
& +C_{F} n_{l}^{3} T_{F}^{3}\left(-\frac{1117984}{2187}-\frac{25984}{81} \zeta_{2}-\frac{1664}{45} \zeta_{2}^{2}-\frac{640}{81} \zeta_{3}\right)+\mathcal{O}(\varepsilon)
\end{aligned}
$$

see also [14], and

$$
\begin{aligned}
G_{S}^{4}= & N_{C}^{4}\left(-\frac{11003395}{4374}+\frac{93937}{972} \zeta_{2}-290 \zeta_{2}^{2}+\frac{12350}{63} \zeta_{2}^{3}-\frac{36592}{9} \zeta_{3}+\frac{1622}{3} \zeta_{3}^{2}+\frac{8480}{9} \zeta_{3} \zeta_{2}-\frac{656}{5} \zeta_{3} \zeta_{2}^{2}+\frac{8780}{3} \zeta_{5}\right. \\
& \left.-288 \zeta_{5} \zeta_{2}-1410 \zeta_{7}\right)+N_{C}^{3} n_{l} T_{F}\left(\frac{14211811}{11664}-\frac{497663}{486} \zeta_{2}+\frac{5027}{15} \zeta_{2}^{2}-\frac{29432}{315} \zeta_{2}^{3}+\frac{199909}{81} \zeta_{3}+\frac{32}{3} \zeta_{3}^{2}-\frac{2432}{9} \zeta_{3} \zeta_{2}\right. \\
& \left.+\frac{620}{9} \zeta_{5}\right)+C_{F}^{2} n_{l}^{2} T_{F}^{2}\left(\frac{201110}{243}-\frac{2944}{9} \zeta_{2}-\frac{16192}{45} \zeta_{2}^{2}+\frac{67360}{27} \zeta_{3}-\frac{512}{3} \zeta_{3} \zeta_{2}-\frac{832}{3} \zeta_{5}\right) \\
& +C_{F} C_{A} n_{l}^{2} T_{F}^{2}\left(-\frac{823055}{1458}+\frac{293144}{243} \zeta_{2}+\frac{2752}{15} \zeta_{2}^{2}-\frac{136544}{81} \zeta_{3}+\frac{1216}{9} \zeta_{3} \zeta_{2}-\frac{4928}{9} \zeta_{5}\right) \\
& +C_{F} n_{l}^{3} T_{F}^{3}\left(-\frac{41728}{2187}-\frac{6400}{81} \zeta_{2}-\frac{1664}{45} \zeta_{2}^{2}-\frac{640}{81} \zeta_{3}\right)+\mathcal{O}(\varepsilon) .
\end{aligned}
$$

Due to the recent two-loop calculations in Ref. [30] we can extract the coefficients in

$$
\mathcal{C}^{(i)}=\sum_{k=0}^{\infty} \varepsilon^{k} \mathcal{C}^{i, k}
$$

up to the required order. They read

$$
\begin{aligned}
\mathcal{C}_{V}^{1,0}= & C_{F}\left(4+\zeta_{2}\right) \\
\mathcal{C}_{V}^{1,1}= & C_{F}\left(8+\frac{1}{2} \zeta_{2}-\frac{2}{3} \zeta_{3}\right), \\
\mathcal{C}_{V}^{1,2}= & C_{F}\left(16+2 \zeta_{2}+\frac{9}{20} \zeta_{2}^{2}-\frac{1}{3} \zeta_{3}\right) \\
\mathcal{C}_{V}^{1,3}= & C_{F}\left(32+4 \zeta_{2}+\frac{9}{40} \zeta_{2}^{2}-\frac{4}{3} \zeta_{3}-\frac{1}{3} \zeta_{2} \zeta_{3}-\frac{2}{5} \zeta_{5}\right), \\
\mathcal{C}_{V}^{2,0}= & C_{F}^{2}\left(\frac{241}{8}+26 \zeta_{2}-48 \ln (2) \zeta_{2}-\frac{163}{10} \zeta_{2}^{2}-6 \zeta_{3}\right)+C_{F} C_{A}\left(\frac{12877}{648}+\frac{323}{18} \zeta_{2}+24 \ln (2) \zeta_{2}-\frac{47}{5} \zeta_{2}^{2}+\frac{89}{9} \zeta_{3}\right) \\
& +C_{F} T_{F} n_{l}\left(-\frac{1541}{162}-\frac{74}{9} \zeta_{2}-\frac{52}{9} \zeta_{3}\right),
\end{aligned}
$$




$$
\begin{aligned}
& \mathcal{C}_{V}^{2,1}=C_{F}^{2}\left(-\frac{557}{16}+8 c_{1}+\frac{399}{4} \zeta_{2}-24 \ln (2) \zeta_{2}-\frac{1363}{10} \zeta_{2}^{2}+\frac{286}{3} \zeta_{3}-\frac{146}{3} \zeta_{2} \zeta_{3}-6 \zeta_{5}\right) \\
& +C_{F} C_{A}\left(\frac{629821}{3888}-4 c_{1}+\frac{7805}{108} \zeta_{2}+12 \ln (2) \zeta_{2}+\frac{209}{2} \zeta_{2}^{2}-\frac{1162}{27} \zeta_{3}+\frac{83}{3} \zeta_{2} \zeta_{3}-208 \zeta_{5}\right) \\
& +C_{F} n_{l} T_{F}\left(-\frac{46205}{972}-\frac{673}{27} \zeta_{2}-\frac{98}{5} \zeta_{2}^{2}-\frac{364}{27} \zeta_{3}\right) \\
& \mathcal{C}_{V}^{2,2}=C_{F}^{2}\left(\frac{1817}{32}+4 c_{1}+\frac{48}{5} c_{2}+8 c_{1} \zeta_{2}+\frac{5165}{8} \zeta_{2}-576 \zeta_{2} \ln (2)+\frac{393}{8} \zeta_{2}^{2}-\frac{37137}{140} \zeta_{2}^{3}-144 \zeta_{2}^{2} \ln ^{2}(2)+\frac{1889}{6} \zeta_{3}\right. \\
& \left.-\frac{799}{9} \zeta_{3}^{2}-\frac{494}{3} \zeta_{3} \zeta_{2}+168 \zeta_{3} \zeta_{2} \ln (2)-1431 \zeta_{5}\right)+C_{F} C_{A}\left(\frac{10899301}{23328}-2 c_{1}-4 c_{1} \zeta_{2}-\frac{24}{5} c_{2}+\frac{53045}{648} \zeta_{2}\right. \\
& \left.+\frac{2513}{24} \zeta_{2}^{2}-\frac{9839}{210} \zeta_{2}^{3}+288 \zeta_{2} \ln (2)+72 \zeta_{2}^{2} \ln ^{2}(2)-\frac{6517}{162} \zeta_{3}+\frac{13}{2} \zeta_{3}^{2}+\frac{1387}{9} \zeta_{3} \zeta_{2}-84 \zeta_{3} \zeta_{2} \ln (2)+\frac{23077}{30} \zeta_{5}\right) \\
& +C_{F} n_{l} T_{F}\left(-\frac{1063589}{5832}-\frac{15481}{162} \zeta_{2}-\frac{1607}{30} \zeta_{2}^{2}-\frac{3422}{81} \zeta_{3}-\frac{224}{9} \zeta_{3} \zeta_{2}-\frac{1444}{15} \zeta_{5}\right) \\
& \mathcal{C}_{V}^{3,0}=2 C_{F}^{2} T_{F} n_{l}\left(-\frac{78427}{972}-\frac{32}{9} c_{1}-\frac{38267}{324} \zeta_{2}+\frac{224}{3} \ln (2) \zeta_{2}+\frac{17873}{135} \zeta_{2}^{2}-\frac{5264}{81} \zeta_{3}-\frac{154}{9} \zeta_{2} \zeta_{3}+\frac{596}{9} \zeta_{5}\right) \\
& +N_{C}^{3}\left(\frac{10907077}{52488}+\frac{15763067}{46656} \zeta_{2}-\frac{116957}{1080} \zeta_{2}^{2}+\frac{145051}{3024} \zeta_{2}^{3}-\frac{15743}{486} \zeta_{3}+\frac{533}{18} \zeta_{2} \zeta_{3}+\frac{298}{9} \zeta_{3}^{2}-357 \zeta_{5}\right) \\
& +2 C_{F} C_{A} T_{F} n_{l}\left(-\frac{533996}{6561}+\frac{16}{9} c_{1}-\frac{102019}{729} \zeta_{2}-\frac{112}{3} \ln (2) \zeta_{2}-\frac{10942}{135} \zeta_{2}^{2}-\frac{2696}{81} \zeta_{3}+\frac{302}{3} \zeta_{5}\right) \\
& +4 C_{F} T_{F}^{2} n_{l}^{2}\left(\frac{58883}{13122}+\frac{580}{81} \zeta_{2}+\frac{884}{135} \zeta_{2}^{2}+\frac{2144}{243} \zeta_{3}\right)
\end{aligned}
$$

$$
\begin{aligned}
& \mathcal{C}_{S}^{1,0}=C_{F} \zeta_{2} \\
& \mathcal{C}_{S}^{1,1}=C_{F}\left(-\zeta_{2}-\frac{2}{3} \zeta_{3}\right) \\
& \mathcal{C}_{S}^{1,2}=C_{F}\left(\frac{9}{20} \zeta_{2}^{2}+\frac{2}{3} \zeta_{3}\right) \\
& \mathcal{C}_{S}^{1,3}=C_{F}\left(-\frac{9}{20} \zeta_{2}^{2}-\frac{1}{3} \zeta_{2} \zeta_{3}-\frac{2}{5} \zeta_{5}\right), \\
& \mathcal{C}_{S}^{2,0}=C_{F}^{2}\left(15-8 \zeta_{2}-\frac{163}{10} \zeta_{2}^{2}-18 \zeta_{3}\right)+C_{F} C_{A}\left(-\frac{2140}{81}+\frac{467}{18} \zeta_{2}-\frac{47}{5} \zeta_{2}^{2}+\frac{143}{9} \zeta_{3}\right)+C_{F} n_{l} T_{F}\left(\frac{188}{81}-\frac{2}{9} \zeta_{2}-\frac{52}{9} \zeta_{3}\right) \\
& \mathcal{C}_{S}^{2,1}=C_{F}^{2}\left(-\frac{209}{2}-76 \zeta_{2}+264 \ln (2) \zeta_{2}-37 \zeta_{2}^{2}-34 \zeta_{3}-\frac{146}{3} \zeta_{2} \zeta_{3}-6 \zeta_{5}\right)+C_{F} C_{A}\left(-\frac{8155}{486}+\frac{2561}{27} \zeta_{2}-132 \ln (2) \zeta_{2}\right. \\
& \left.+\frac{541}{10} \zeta_{2}^{2}+\frac{242}{27} \zeta_{3}+\frac{83}{3} \zeta_{2} \zeta_{3}-208 \zeta_{5}\right)+C_{F} n_{l} T_{F}\left(\frac{214}{243}+\frac{200}{27} \zeta_{2}-\frac{98}{5} \zeta_{2}^{2}+\frac{68}{27} \zeta_{3}\right) \\
& \mathcal{C}_{S}^{2,2}=C_{F}^{2}\left(-\frac{779}{4}-44 c_{1}+8 c_{1} \zeta_{2}-17 \zeta_{2}+480 \zeta_{2} \ln (2)+\frac{857}{2} \zeta_{2}^{2}-\frac{37137}{140} \zeta_{2}^{3}-144 \zeta_{2}^{2} \ln ^{2}(2)-272 \zeta_{3}-\frac{799}{9} \zeta_{3}^{2}\right. \\
& \left.-\frac{92}{3} \zeta_{3} \zeta_{2}+168 \zeta_{3} \zeta_{2} \ln (2)-213 \zeta_{5}\right)+C_{F} C_{A}\left(-\frac{415831}{2916}+22 c_{1}-4 c_{1} \zeta_{2}+\frac{11818}{81} \zeta_{2}-240 \zeta_{2} \ln (2)\right. \\
& \left.-\frac{2098}{15} \zeta_{2}^{2}-\frac{9839}{210} \zeta_{2}^{3}+72 \zeta_{2}^{2} \ln ^{2}(2)+\frac{14422}{81} \zeta_{3}+\frac{13}{2} \zeta_{3}^{2}+\frac{793}{9} \zeta_{3} \zeta_{2}-84 \zeta_{3} \zeta_{2} \ln (2)+\frac{4807}{30} \zeta_{5}\right) \\
& +C_{F} n_{l} T_{F}\left(-\frac{9049}{729}+\frac{940}{81} \zeta_{2}+\frac{92}{15} \zeta_{2}^{2}+\frac{880}{81} \zeta_{3}-\frac{224}{9} \zeta_{3} \zeta_{2}-\frac{1444}{15} \zeta_{5}\right)
\end{aligned}
$$




$$
\begin{aligned}
\mathcal{C}_{S}^{3,0}= & N_{C}^{3}\left(-\frac{10407317}{52488}+\frac{324905}{2916} \zeta_{2}-\frac{77213}{1080} \zeta_{2}^{2}+\frac{145051}{3024} \zeta_{2}^{3}-\frac{28865}{486} \zeta_{3}+\frac{1181}{18} \zeta_{2} \zeta_{3}+\frac{298}{9} \zeta_{3}^{2}-372 \zeta_{5}\right) \\
& +2 C_{F}^{2} T_{F} n_{l}\left(\frac{32351}{972}+\frac{9545}{162} \zeta_{2}-160 \ln (2) \zeta_{2}+\frac{1969}{27} \zeta_{2}^{2}+\frac{1612}{81} \zeta_{3}-\frac{154}{9} \zeta_{2} \zeta_{3}+\frac{596}{9} \zeta_{5}\right) \\
& +2 C_{F} C_{A} T_{F} n_{l}\left(\frac{842609}{13122}-\frac{45319}{729} \zeta_{2}+80 \ln (2) \zeta_{2}-\frac{9574}{135} \zeta_{2}^{2}-\frac{1094}{81} \zeta_{3}+\frac{302}{3} \zeta_{5}\right) \\
& +4 C_{F} T_{F}^{2} n_{l}^{2}\left(-\frac{2324}{6561}-\frac{356}{81} \zeta_{2}+\frac{884}{135} \zeta_{2}^{2}+\frac{632}{243} \zeta_{3}\right) .
\end{aligned}
$$

The functions $\mathcal{C}_{V}^{3,0}$ and $\mathcal{C}_{S}^{3,0}$ have been obtained from the results in [31-34]. Here $\mathcal{C}_{V}$ and $\mathcal{C}_{S}$ have been calculated individually. One may check that they are related by

$$
\mathcal{C}_{S}=\frac{Z_{m, \mathrm{OS}}}{Z_{m, \overline{\mathrm{MS}}}} \mathcal{C}_{V}
$$

With all the available components, we can now present all the logarithmic contributions to the finite part for three-loop vector and scalar form factors in the asymptotic limit:

$$
\begin{aligned}
& F_{V}^{(3)}=\frac{1}{\varepsilon^{3}}\left[-C_{F}^{3} \frac{4}{3}(1-L)^{3}-C_{F}^{2} C_{A} \frac{22}{3}(1-L)^{2}-C_{F} C_{A}^{2} \frac{242}{27}(1-L)+C_{F} C_{A} n_{l} T_{F} \frac{176}{27}(1-L)\right. \\
& \left.+C_{F}^{2} n_{l} T_{F} \frac{8}{3}(1-L)^{2}-C_{F} n_{l}^{2} T_{F}^{2} \frac{32}{27}(1-L)\right]+\frac{1}{\varepsilon^{2}}\left[C _ { F } ^ { 3 } \left\{-8+4 \zeta_{2}+\left(22-8 \zeta_{2}\right) L+\left(-22+4 \zeta_{2}\right) L^{2}\right.\right. \\
& \left.+10 L^{3}-2 L^{4}\right\}+C_{F}^{2} C_{A}\left\{-\frac{34}{9}+\frac{10}{3} \zeta_{2}+4 \zeta_{3}+\left(-\frac{1}{9}+\frac{2 \zeta_{2}}{3}-4 \zeta_{3}\right) L+\left(\frac{2}{9}-4 \zeta_{2}\right) L^{2}+\frac{11 L^{3}}{3}\right\} \\
& +C_{F} C_{A}^{2}\left\{\frac{1690}{81}-\frac{44}{9} \zeta_{2}+\frac{44}{9} \zeta_{3}+\left(-\frac{2086}{81}+\frac{44 \zeta_{2}}{9}\right) L\right\}+C_{F} C_{A} n_{l} T_{F}\left\{-\frac{1192}{81}+\frac{16}{9} \zeta_{2}-\frac{16}{9} \zeta_{3}\right. \\
& \left.\left.+\left(\frac{1336}{81}-\frac{16 \zeta_{2}}{9}\right) L\right\}+C_{F}^{2} n_{l} T_{F}\left\{-\frac{16}{9}-\frac{8}{3} \zeta_{2}+\left(\frac{20}{9}+\frac{8 \zeta_{2}}{3}\right) L+\frac{8 L^{2}}{9}-\frac{4 L^{3}}{3}\right\}+C_{F} n_{l}^{2} T_{F}^{2} \frac{160}{81}(1-L)\right] \\
& +\frac{1}{\varepsilon}\left[C _ { F } ^ { 3 } \left\{-76-82 \zeta_{2}+96 \ln (2) \zeta_{2}+\frac{236}{5} \zeta_{2}^{2}+80 \zeta_{3}+\left(129+88 \zeta_{2}-96 \ln (2) \zeta_{2}-\frac{236}{5} \zeta_{2}^{2}-136 \zeta_{3}\right) L\right.\right. \\
& \left.+\left(-89+56 \zeta_{3}\right) L^{2}+\left(\frac{137}{3}-6 \zeta_{2}\right) L^{3}-\frac{34 L^{4}}{3}+\frac{5 L^{5}}{3}\right\}+C_{F}^{2} C_{A}\left\{\frac{2986}{27}-10 \zeta_{2}-48 \ln (2) \zeta_{2}+\frac{26}{5} \zeta_{2}^{2}\right. \\
& -\frac{200}{3} \zeta_{3}-4 \zeta_{2} \zeta_{3}+\left(-\frac{5396}{27}+\frac{5}{3} \zeta_{2}+48 \ln (2) \zeta_{2}-\frac{26}{5} \zeta_{2}^{2}+\frac{362}{3} \zeta_{3}\right) L+\left(\frac{6107}{54}+\frac{19 \zeta_{2}}{3}-50 \zeta_{3}\right) L^{2} \\
& \left.+\left(-\frac{523}{18}+6 \zeta_{2}\right) L^{3}+\frac{11 L^{4}}{9}\right\}+C_{F} C_{A}^{2}\left\{-\frac{343}{27}+\frac{608}{27} \zeta_{2}-\frac{88}{15} \zeta_{2}^{2}-\frac{740}{27} \zeta_{3}-\frac{8}{3} \zeta_{2} \zeta_{3}+12 \zeta_{5}\right. \\
& \left.+\left(\frac{245}{9}-\frac{536}{27} \zeta_{2}+\frac{88}{15} \zeta_{2}^{2}+\frac{44}{9} \zeta_{3}\right) L\right\}+C_{F} C_{A} n_{l} T_{F}\left\{\frac{356}{81}-\frac{160}{27} \zeta_{2}+\frac{496}{27} \zeta_{3}+\left(-\frac{836}{81}+\frac{160 \zeta_{2}}{27}-\frac{112 \zeta_{3}}{9}\right) L\right\} \\
& +C_{F}^{2} n_{l} T_{F}\left\{-\frac{470}{27}+8 \zeta_{2}-\frac{16}{3} \zeta_{3}+\left(\frac{1198}{27}-\frac{4 \zeta_{2}}{3}+\frac{16 \zeta_{3}}{3}\right) L+\left(-\frac{962}{27}-\frac{20 \zeta_{2}}{3}\right) L^{2}+\frac{82 L^{3}}{9}-\frac{4 L^{4}}{9}\right\} \\
& \left.+C_{F} n_{l}^{2} T_{F}^{2} \frac{32}{81}(1-L)\right]+\left[X_{V, 3}^{0,0}+N_{C}^{3}\left\{-\frac{554267}{2916}+\frac{23773 \zeta_{2}}{216}-\frac{1727 \zeta_{2}^{2}}{30}+\frac{8156 \zeta_{2}^{3}}{315}+\frac{33197 \zeta_{3}}{162}-\frac{16 \zeta_{3}^{2}}{3}\right.\right. \\
& \left.+\frac{113 \zeta_{3} \zeta_{2}}{3}-\frac{875 \zeta_{5}}{3}\right\}+C_{F}^{3}\left\{\left(250+16 c_{1}+585 \zeta_{2}-192 \ln (2) \zeta_{2}-\frac{1942}{5} \zeta_{2}^{2}-520 \zeta_{3}-8 \zeta_{2} \zeta_{3}-276 \zeta_{5}\right) L\right. \\
& \left.-\left(332+123 \zeta_{2}-48 \ln (2) \zeta_{2}-\frac{302}{5} \zeta_{2}^{2}-340 \zeta_{3}\right) L^{2}+\left(\frac{494}{3}+\frac{17 \zeta_{2}}{3}-\frac{268 \zeta_{3}}{3}\right) L^{3}-\left(\frac{148}{3}-\frac{16 \zeta_{2}}{3}\right) L^{4}+\frac{17 L^{5}}{2}-L^{6}\right\}
\end{aligned}
$$




$$
\begin{aligned}
& +C_{F}^{2} C_{A}\left\{\left(-\frac{178337}{162}-8 c_{1}-\frac{5959}{18} \zeta_{2}+96 \ln (2) \zeta_{2}+\frac{4279}{15} \zeta_{2}^{2}+\frac{3706}{3} \zeta_{3}-46 \zeta_{2} \zeta_{3}-194 \zeta_{5}\right) L\right. \\
& +\left(\frac{64625}{81}+\frac{266}{3} \zeta_{2}-24 \ln (2) \zeta_{2}-\frac{143}{5} \zeta_{2}^{2}-\frac{3743}{9} \zeta_{3}\right) L^{2}+\left(-\frac{6260}{27}-\frac{97 \zeta_{2}}{18}+\frac{232 \zeta_{3}}{3}\right) L^{3} \\
& \left.+\left(\frac{4289}{108}-\frac{16 \zeta_{2}}{3}\right) L^{4}-\frac{11 L^{5}}{4}\right\}+C_{F} C_{A}^{2}\left\{\left(\frac{1045955}{1458}+\frac{17366}{81} \zeta_{2}-\frac{94}{3} \zeta_{2}^{2}-\frac{17464}{27} \zeta_{3}+\frac{88}{3} \zeta_{2} \zeta_{3}+136 \zeta_{5}\right) L\right. \\
& \left.+\left(-\frac{18682}{81}+\frac{26}{9} \zeta_{2}-\frac{44}{5} \zeta_{2}^{2}+88 \zeta_{3}\right) L^{2}+\left(\frac{2869}{81}-\frac{44 \zeta_{2}}{9}\right) L^{3}-\frac{121 L^{4}}{54}\right\}+C_{F} C_{A} n_{l} T_{F}\left\{\frac{259150}{729}+\frac{32 c_{1}}{9}\right. \\
& +\frac{3008 \zeta_{2}}{81}-\frac{7288 \zeta_{2}^{2}}{45}-\frac{224}{3} \zeta_{2} \ln (2)-\frac{31120 \zeta_{3}}{81}+\frac{8 \zeta_{3} \zeta_{2}}{3}+\frac{596 \zeta_{5}}{3}+\left(-\frac{309838}{729}-\frac{11728}{81} \zeta_{2}+\frac{88}{15} \zeta_{2}^{2}+\frac{1448}{9} \zeta_{3}\right) L \\
& \left.+\left(\frac{11752}{81}+\frac{32 \zeta_{2}}{3}-16 \zeta_{3}\right) L^{2}+\left(-\frac{1948}{81}+\frac{16 \zeta_{2}}{9}\right) L^{3}+\frac{44 L^{4}}{27}\right\}+C_{F}^{2} n_{l} T_{F}\left\{-\frac{2011}{81}-\frac{64 c_{1}}{9}-\frac{962 \zeta_{2}}{3}\right. \\
& +\frac{12232 \zeta_{2}^{2}}{45}+\frac{2752 \zeta_{3}}{9}-48 \zeta_{3} \zeta_{2}+40 \zeta_{5}+\frac{448 \zeta_{2} \ln (2)}{3}+\left(\frac{18812}{81}+\frac{682}{9} \zeta_{2}-\frac{392}{15} \zeta_{2}^{2}-\frac{1976}{9} \zeta_{3}\right) L \\
& \left.+\left(-\frac{18817}{81}-\frac{100 \zeta_{2}}{3}+\frac{232 \zeta_{3}}{9}\right) L^{2}+\left(\frac{2032}{27}+\frac{58 \zeta_{2}}{9}\right) L^{3}-\frac{355 L^{4}}{27}+L^{5}\right\}+C_{F} n_{l}^{2} T_{F}^{2}\left\{-\frac{29344}{729}-\frac{976 \zeta_{2}}{81}\right. \\
& \left.\left.+\frac{928 \zeta_{2}^{2}}{45}+\frac{256 \zeta_{3}}{9}+\left(\frac{39352}{729}+\frac{608 \zeta_{2}}{27}+\frac{64 \zeta_{3}}{27}\right) L-\left(\frac{1624}{81}+\frac{32 \zeta_{2}}{9}\right) L^{2}+\frac{304 L^{3}}{81}-\frac{8 L^{4}}{27}\right\}\right] \text {. } \\
& F_{S}^{(3)}=\frac{1}{\varepsilon^{3}}\left[-C_{F}^{3} \frac{4}{3}(1-L)^{3}-C_{F}^{2} C_{A} \frac{22}{3}(1-L)^{2}-C_{F} C_{A}^{2} \frac{242}{27}(1-L)+C_{F} C_{A} n_{l} T_{F} \frac{176}{27}(1-L)+C_{F}^{2} n_{l} T_{F} \frac{8}{3}(1-L)^{2}\right. \\
& \left.-C_{F} n_{l}^{2} T_{F}^{2} \frac{32}{27}(1-L)\right]+\frac{1}{\varepsilon^{2}}\left[C_{F}^{3}\left\{-4+4 \zeta_{2}+\left(8-8 \zeta_{2}\right) L+\left(-6+4 \zeta_{2}\right) L^{2}+4 L^{3}-2 L^{4}\right\}\right. \\
& +C_{F}^{2} C_{A}\left\{\frac{32}{9}+\frac{10}{3} \zeta_{2}+4 \zeta_{3}+\left(-\frac{166}{9}+\frac{2 \zeta_{2}}{3}-4 \zeta_{3}\right) L+\left(\frac{101}{9}-4 \zeta_{2}\right) L^{2}+\frac{11 L^{3}}{3}\right\} \\
& +C_{F} C_{A}^{2}\left\{\frac{1690}{81}+\frac{44}{9}\left(\zeta_{3}-\zeta_{2}\right)+\left(-\frac{2086}{81}+\frac{44 \zeta_{2}}{9}\right) L\right\}+C_{F} C_{A} n_{l} T_{F}\left\{-\frac{1192}{81}+\frac{16}{9} \zeta_{2}-\frac{16}{9} \zeta_{3}\right. \\
& \left.\left.+\left(\frac{1336}{81}-\frac{16 \zeta_{2}}{9}\right) L\right\}+C_{F}^{2} n_{l} T_{F}\left\{-\frac{40}{9}-\frac{8}{3} \zeta_{2}+\left(\frac{80}{9}+\frac{8 \zeta_{2}}{3}\right) L-\frac{28 L^{2}}{9}-\frac{4 L^{3}}{3}\right\}+C_{F} n_{l}^{2} T_{F}^{2} \frac{160}{81}(1-L)\right] \\
& +\frac{1}{\varepsilon}\left[C _ { F } ^ { 3 } \left\{-50-10 \zeta_{2}+\frac{236}{5} \zeta_{2}^{2}+104 \zeta_{3}+\left(62+34 \zeta_{2}-\frac{236}{5} \zeta_{2}^{2}-160 \zeta_{3}\right) L-\left(20+18 \zeta_{2}-56 \zeta_{3}\right) L^{2}\right.\right. \\
& \left.+\left(\frac{26}{3}-6 \zeta_{2}\right) L^{3}-\frac{7 L^{4}}{3}+\frac{5 L^{5}}{3}\right\}+C_{F}^{2} C_{A}\left\{\frac{1636}{27}-59 \zeta_{2}+\frac{26}{5} \zeta_{2}^{2}-\frac{140}{3} \zeta_{3}-4 \zeta_{2} \zeta_{3}\right. \\
& \left.+\left(-\frac{2030}{27}+\frac{134}{3} \zeta_{2}-\frac{26}{5} \zeta_{2}^{2}+\frac{308}{3} \zeta_{3}\right) L+\left(\frac{835}{27}+\frac{37 \zeta_{2}}{3}-50 \zeta_{3}\right) L^{2}+\left(-\frac{212}{9}+6 \zeta_{2}\right) L^{3}+\frac{11 L^{4}}{9}\right\} \\
& +C_{F} C_{A}^{2}\left\{-\frac{343}{27}+\frac{608}{27} \zeta_{2}-\frac{88}{15} \zeta_{2}^{2}-\frac{740}{27} \zeta_{3}-\frac{8}{3} \zeta_{2} \zeta_{3}+12 \zeta_{5}+\left(\frac{245}{9}-\frac{536}{27} \zeta_{2}+\frac{88}{15} \zeta_{2}^{2}+\frac{44}{9} \zeta_{3}\right) L\right\} \\
& +C_{F} C_{A} n_{l} T_{F}\left\{\frac{356}{81}-\frac{160}{27} \zeta_{2}+\frac{496}{27} \zeta_{3}+\left(-\frac{836}{81}+\frac{160 \zeta_{2}}{27}-\frac{112 \zeta_{3}}{9}\right) L\right\}+C_{F}^{2} n_{l} T_{F}\left\{-\frac{38}{27}+4 \zeta_{2}-\frac{16}{3} \zeta_{3}\right. \\
& \left.\left.+\left(\frac{190}{27}+\frac{8 \zeta_{2}}{3}+\frac{16 \zeta_{3}}{3}\right) L+\left(-\frac{332}{27}-\frac{20 \zeta_{2}}{3}\right) L^{2}+\frac{64 L^{3}}{9}-\frac{4 L^{4}}{9}\right\}+C_{F} n_{l}^{2} T_{F}^{2} \frac{32}{81}(1-L)\right] \\
& +\left[X_{S, 3}^{0,0}+N_{C}^{3}\left\{-\frac{133949}{2916}+\frac{6623 \zeta_{2}}{108}-\frac{545 \zeta_{2}^{2}}{12}+\frac{8156 \zeta_{2}^{3}}{315}+\frac{3403 \zeta_{3}}{324}-\frac{16 \zeta_{3}^{2}}{3}+\frac{397 \zeta_{3} \zeta_{2}}{6}-\frac{830 \zeta_{5}}{3}\right\}\right.
\end{aligned}
$$




$$
\begin{aligned}
& +C_{F}^{3}\left\{\left(-131+14 \zeta_{2}+528 \ln (2) \zeta_{2}-\frac{616}{5} \zeta_{2}^{2}-428 \zeta_{3}-8 \zeta_{2} \zeta_{3}-276 \zeta_{5}\right) L-\left(87+79 \zeta_{2}-\frac{302}{5} \zeta_{2}^{2}-244 \zeta_{3}\right) L^{2}\right. \\
& \left.+\left(\frac{76}{3}+\frac{98 \zeta_{2}}{3}-\frac{268 \zeta_{3}}{3}\right) L^{3}+\left(-\frac{17}{2}+\frac{16 \zeta_{2}}{3}\right) L^{4}+L^{5}-L^{6}\right\}+C_{F}^{2} C_{A}\left\{\left(-\frac{8569}{81}-\frac{1091}{9} \zeta_{2}-264 \ln (2) \zeta_{2}\right.\right. \\
& \left.+\frac{2524}{15} \zeta_{2}^{2}+\frac{2686}{3} \zeta_{3}-46 \zeta_{2} \zeta_{3}-194 \zeta_{5}\right) L+\left(\frac{9824}{81}+\frac{175}{6} \zeta_{2}-\frac{143}{5} \zeta_{2}^{2}-\frac{2312}{9} \zeta_{3}\right) L^{2}+\left(-\frac{1208}{27}-\frac{259 \zeta_{2}}{18}+\frac{232 \zeta_{3}}{3}\right) L^{3} \\
& \left.+\left(\frac{2309}{108}-\frac{16 \zeta_{2}}{3}\right) L^{4}-\frac{11 L^{5}}{4}\right\}+C_{F} C_{A}^{2}\left\{\left(\frac{10289}{1458}+\frac{9644}{81} \zeta_{2}-\frac{94}{3} \zeta_{2}^{2}-\frac{13900}{27} \zeta_{3}+\frac{88}{3} \zeta_{2} \zeta_{3}+136 \zeta_{5}\right) L\right. \\
& \left.+\left(-\frac{11939}{162}+\frac{26}{9} \zeta_{2}-\frac{44}{5} \zeta_{2}^{2}+88 \zeta_{3}\right) L^{2}+\left(\frac{1780}{81}-\frac{44 \zeta_{2}}{9}\right) L^{3}-\frac{121 L^{4}}{54}\right\}+C_{F} C_{A} n_{l} T_{F}\left\{\frac{35626}{729}-\frac{2680 \zeta_{2}}{81}\right. \\
& -\frac{1340 \zeta_{2}^{2}}{9}+160 \zeta_{2} \ln (2)-\frac{19348 \zeta_{3}}{81}+\frac{8 \zeta_{3} \zeta_{2}}{3}+\frac{596 \zeta_{5}}{3}+\left(-\frac{14998}{729}-\frac{6544}{81} \zeta_{2}+\frac{88}{15} \zeta_{2}^{2}+\frac{1448}{9} \zeta_{3}\right) L \\
& \left.+\left(\frac{3454}{81}+\frac{32 \zeta_{2}}{3}-16 \zeta_{3}\right) L^{2}+\left(-\frac{1156}{81}+\frac{16 \zeta_{2}}{9}\right) L^{3}+\frac{44 L^{4}}{27}\right\}+C_{F}^{2} n_{l} T_{F}\left\{\frac{7727}{81}+22 \zeta_{2}+\frac{1592}{9} \zeta_{2}^{2}-320 \zeta_{2} \ln (2)\right. \\
& +\frac{3328}{9} \zeta_{3}-48 \zeta_{3} \zeta_{2}+40 \zeta_{5}+\left(\frac{1415}{81}+\frac{820}{9} \zeta_{2}-\frac{392}{15} \zeta_{2}^{2}-\frac{1904}{9} \zeta_{3}\right) L+\left(-\frac{2635}{81}-\frac{58 \zeta_{2}}{3}+\frac{232 \zeta_{3}}{9}\right) L^{2} \\
& \left.+\left(\frac{460}{27}+\frac{58 \zeta_{2}}{9}\right) L^{3}-\frac{175 L^{4}}{27}+L^{5}\right\}+C_{F} n_{l}^{2} T_{F}^{2}\left\{-\frac{112}{729}-\frac{2272 \zeta_{2}}{81}+\frac{928 \zeta_{2}^{2}}{45}+\frac{64 \zeta_{3}}{9}+\left(\frac{3712}{729}+\frac{320 \zeta_{2}}{27}+\frac{64 \zeta_{3}}{27}\right) L\right. \\
& \left.\left.+\left(-\frac{400}{81}-\frac{32 \zeta_{2}}{9}\right) L^{2}+\frac{160 L^{3}}{81}-\frac{8 L^{4}}{27}\right\}\right] .
\end{aligned}
$$

Here we used the abbreviation

$$
c_{1}=12 \zeta_{2} \ln ^{2}(2)+\ln ^{4}(2)+24 \mathrm{Li}_{4}\left(\frac{1}{2}\right)
$$

where $\operatorname{Li}_{k}(x)$ denotes the polylogarithm [66-68]. The functions $X_{V, 3}^{0,0}$ and $X_{S, 3}^{0,0}$ are independent of $L$ and contain the subleading contributions in $N_{C}$ which can be obtained through an exact calculation only, including the nonplanar color topologies. The leading pole contributions are the same in the vector and scalar cases, also for the four-loop terms given below.

At the four-loop level, we obtain the complete contributions to the $\frac{1}{\varepsilon^{4}}, \frac{1}{\varepsilon^{3}}$, and $\frac{1}{\varepsilon^{2}}$ poles, leading color contributions to $L$ and complete $L^{2} \ldots L^{\mathrm{Max}}$ of the single pole in $\varepsilon$ and leading color contributions to $L^{2}$ and complete $L^{3} \ldots L^{\mathrm{Max}}$ for the finite pieces. The yet unknown terms are denoted by $X_{I, n}^{k, j}$ indicating the $\varepsilon^{k} L^{j}$-coefficient of $F_{I}^{(n)}$. Again the terms $X_{I, n}^{k, j}$ do not contain logarithmic contributions.

A partial prediction of the four-loop vector and scalar form factor is given by

$$
\begin{aligned}
F_{V}^{(4)}= & \frac{1}{\varepsilon^{4}}\left[C_{F}^{4} \frac{2}{3}(1-L)^{4}+C_{A} C_{F}^{3} \frac{22}{3}(1-L)^{3}+C_{A}^{2} C_{F}^{2} \frac{1331}{54}(1-L)^{2}+C_{A}^{3} C_{F} \frac{1331}{54}(1-L)-C_{F}^{3} n_{l} T_{F} \frac{8}{3}(1-L)^{3}\right. \\
& -C_{A} C_{F}^{2} n_{l} T_{F} \frac{484}{27}(1-L)^{2}-C_{A}^{2} C_{F} n_{l} T_{F} \frac{242}{9}(1-L)+C_{F}^{2} n_{l}^{2} T_{F}^{2} \frac{88}{27}(1-L)^{2}+C_{A} C_{F} n_{l}^{2} T_{F}^{2} \frac{88}{9}(1-L) \\
& \left.-C_{F} n_{l}^{3} T_{F}^{3} \frac{32}{27}(1-L)\right]+\frac{1}{\varepsilon^{3}}\left[C _ { F } ^ { 4 } \left\{\left(\frac{16}{3}-\frac{8}{3} \zeta_{2}\right)+\left(-20+8 \zeta_{2}\right) L+\left(\frac{88}{3}-8 \zeta_{2}\right) L^{2}+\left(-\frac{64}{3}+\frac{8}{3} \zeta_{2}\right) L^{3}\right.\right. \\
& \left.+8 L^{4}-\frac{4}{3} L^{5}\right\}+C_{A} C_{F}^{3}\left\{\left(\frac{166}{9}-4 \zeta_{3}-\frac{32}{3} \zeta_{2}\right)+\left(-44+8 \zeta_{3}+\frac{52}{3} \zeta_{2}\right) L+\left(40-4 \zeta_{3}-\frac{8}{3} \zeta_{2}\right) L^{2}\right.
\end{aligned}
$$




$$
\begin{aligned}
& \left.-\left(\frac{196}{9}+4 \zeta_{2}\right) L^{3}+\frac{22}{3} L^{4}\right\}+C_{A}^{2} C_{F}^{2}\left\{\left(-\frac{2093}{81}-\frac{154}{9} \zeta_{3}-\frac{22}{27} \zeta_{2}\right)+\left(\frac{6298}{81}+\frac{154}{9} \zeta_{3}-\frac{440}{27} \zeta_{2}\right) L\right. \\
& \left.+\left(-\frac{3479}{81}+\frac{154}{9} \zeta_{2}\right) L^{2}-\frac{242}{27} L^{3}\right\}+C_{A}^{3} C_{F}\left\{\left(-\frac{12661}{162}-\frac{121}{9} \zeta_{3}+\frac{121}{9} \zeta_{2}\right)+\left(\frac{14839}{162}-\frac{121}{9} \zeta_{2}\right) L\right\} \\
& +C_{F}^{3} n_{l} T_{F}\left\{\left(-\frac{8}{9}+\frac{16}{3} \zeta_{2}\right)+\left(\frac{16}{3}-\frac{32}{3} \zeta_{2}\right) L-\left(\frac{32}{3}-\frac{16}{3} \zeta_{2}\right) L^{2}+\frac{80}{9} L^{3}-\frac{8}{3} L^{4}\right\} \\
& +C_{A} C_{F}^{2} n_{l} T_{F}\left\{\left(\frac{2708}{81}+\frac{56}{9} \zeta_{3}+\frac{184}{27} \zeta_{2}\right)+\left(-\frac{5260}{81}-\frac{56}{9} \zeta_{3}-\frac{16}{27} \zeta_{2}\right) L+\left(\frac{2024}{81}-\frac{56}{9} \zeta_{2}\right) L^{2}+\frac{176}{27} L^{3}\right\} \\
& +C_{A}^{2} C_{F} n_{l} T_{F}\left\{\left(\frac{730}{9}+\frac{88}{9} \zeta_{3}-\frac{88}{9} \zeta_{2}\right)+\left(-\frac{818}{9}+\frac{88}{9} \zeta_{2}\right) L\right\}+C_{F}^{2} n_{l}^{2} T_{F}^{2}\left\{\left(-\frac{608}{81}-\frac{64}{27} \zeta_{2}\right)+\left(\frac{880}{81}+\frac{64}{27} \zeta_{2}\right) L\right. \\
& \left.\left.-\frac{176}{81} L^{2}-\frac{32}{27} L^{3}\right\}+C_{A} C_{F} n_{l}^{2} T_{F}^{2}\left\{\left(-\frac{664}{27}-\frac{16}{9} \zeta_{3}+\frac{16}{9} \zeta_{2}\right)+\left(\frac{712}{27}-\frac{16}{9} \zeta_{2}\right) L\right\}+C_{F} n_{l}^{3} T_{F}^{3} \frac{160}{81}(1-L)\right] \\
& +\frac{1}{\varepsilon^{2}}\left[C _ { F } ^ { 4 } \left\{\frac{212}{3}-\frac{232}{3} \zeta_{3}+\frac{250}{3} \zeta_{2}-\frac{236}{5} \zeta_{2}^{2}-96 \ln (2) \zeta_{2}+\left(-\frac{551}{3}+208 \zeta_{3}-\frac{524}{3} \zeta_{2}+\frac{472}{5} \zeta_{2}^{2}+192 \ln (2) \zeta_{2}\right) L\right.\right. \\
& +\left(185-184 \zeta_{3}+94 \zeta_{2}-\frac{236}{5} \zeta_{2}^{2}-96 \ln (2) \zeta_{2}\right) L^{2}+\left(-\frac{991}{9}+\frac{160}{3} \zeta_{3}+\frac{8}{3} \zeta_{2}\right) L^{3}+\left(48-\frac{16}{3} \zeta_{2}\right) L^{4} \\
& \left.-\frac{34}{3} L^{5}+\frac{13}{9} L^{6}\right\}+C_{A} C_{F}^{3}\left\{\frac{188}{27}-88 \zeta_{3}+\frac{1613}{9} \zeta_{2}+8 \zeta_{2} \zeta_{3}-\frac{1436}{15} \zeta_{2}^{2}-128 \ln (2) \zeta_{2}+\left(\frac{851}{6}+76 \zeta_{3}-\frac{1987}{9} \zeta_{2}\right.\right. \\
& \left.-8 \zeta_{2} \zeta_{3}+\frac{1574}{15} \zeta_{2}^{2}+80 \ln (2) \zeta_{2}\right) L+\left(-\frac{671}{3}+60 \zeta_{3}+\frac{290}{9} \zeta_{2}-\frac{46}{5} \zeta_{2}^{2}+48 \ln (2) \zeta_{2}\right) L^{2} \\
& \left.+\left(\frac{5057}{54}-48 \zeta_{3}+\frac{4}{3} \zeta_{2}\right) L^{3}+\left(-\frac{305}{18}+8 \zeta_{2}\right) L^{4}-\frac{11}{6} L^{5}\right\}+C_{A}^{2} C_{F}^{2}\left\{-\frac{10159}{54}+\frac{4700}{27} \zeta_{3}+2 \zeta_{3}^{2}-\frac{1249}{81} \zeta_{2}\right. \\
& +\frac{100}{9} \zeta_{2} \zeta_{3}+\frac{79}{45} \zeta_{2}^{2}+88 \ln (2) \zeta_{2}-24 \zeta_{5}+\left(\frac{47179}{162}-\frac{7778}{27} \zeta_{3}+\frac{6595}{81} \zeta_{2}-\frac{4}{3} \zeta_{2} \zeta_{3}-\frac{697}{45} \zeta_{2}^{2}-88 \ln (2) \zeta_{2}+24 \zeta_{5}\right) L \\
& \left.+\left(-\frac{8159}{54}+\frac{902}{9} \zeta_{3}-\frac{572}{9} \zeta_{2}+\frac{206}{15} \zeta_{2}^{2}\right) L^{2}+\left(\frac{9925}{162}-\frac{110}{9} \zeta_{2}\right) L^{3}-\frac{121}{81} L^{4}\right\}+C_{A}^{3} C_{F}\left\{\frac{4981}{54}+\frac{2341}{27} \zeta_{3}\right. \\
& \left.-\frac{1978}{27} \zeta_{2}+\frac{22}{3} \zeta_{2} \zeta_{3}+\frac{242}{15} \zeta_{2}^{2}-33 \zeta_{5}+\left(-\frac{287}{2}-\frac{121}{9} \zeta_{3}+\frac{1780}{27} \zeta_{2}-\frac{242}{15} \zeta_{2}^{2}\right) L\right\}+C_{F}^{3} n_{l} T_{F}\left\{-\frac{673}{27}+\frac{208}{3} \zeta_{3}\right. \\
& -\frac{652}{9} \zeta_{2}+\frac{472}{15} \zeta_{2}^{2}+64 \ln (2) \zeta_{2}+\left(\frac{41}{9}-\frac{368}{3} \zeta_{3}+\frac{740}{9} \zeta_{2}-\frac{472}{15} \zeta_{2}^{2}-64 \ln (2) \zeta_{2}\right) L+\left(\frac{392}{9}+\frac{160}{3} \zeta_{3}+\frac{8}{9} \zeta_{2}\right) L^{2} \\
& \left.+\left(-\frac{758}{27}-\frac{32}{3} \zeta_{2}\right) L^{3}+\frac{38}{9} L^{4}+\frac{2}{3} L^{5}\right\}+C_{A} C_{F}^{2} n_{l} T_{F}\left\{\frac{5369}{81}-\frac{556}{9} \zeta_{3}-\frac{1100}{81} \zeta_{2}-\frac{32}{9} \zeta_{2} \zeta_{3}+\frac{196}{45} \zeta_{2}^{2}-32 \ln (2) \zeta_{2}\right. \\
& +\left(-\frac{3883}{27}+\frac{1072}{9} \zeta_{3}-\frac{1612}{81} \zeta_{2}-\frac{196}{45} \zeta_{2}^{2}+32 \ln (2) \zeta_{2}\right) L+\left(\frac{9512}{81}-\frac{520}{9} \zeta_{3}+\frac{880}{27} \zeta_{2}\right) L^{2}+\left(-\frac{3284}{81}+\frac{40}{9} \zeta_{2}\right) L^{3} \\
& \left.+\frac{88}{81} L^{4}\right\}+C_{A}^{2} C_{F} n_{l} T_{F}\left\{-\frac{13241}{162}-\frac{2284}{27} \zeta_{3}+\frac{1228}{27} \zeta_{2}-\frac{8}{3} \zeta_{2} \zeta_{3}-\frac{88}{15} \zeta_{2}^{2}+12 \zeta_{5}+\left(\frac{19313}{162}+\frac{352}{9} \zeta_{3}-\frac{1156}{27} \zeta_{2}\right.\right. \\
& \left.\left.+\frac{88}{15} \zeta_{2}^{2}\right) L\right\}+C_{F}^{2} n_{l}^{2} T_{F}^{2}\left\{\frac{116}{81}-\frac{224}{27} \zeta_{3}+\frac{464}{81} \zeta_{2}+\left(\frac{1132}{81}+\frac{224}{27} \zeta_{3}-\frac{80}{81} \zeta_{2}\right) L+\left(-\frac{1720}{81}-\frac{128}{27} \zeta_{2}\right) L^{2}\right. \\
& \left.+\frac{488}{81} L^{3}-\frac{16}{81} L^{4}\right\}+C_{A} C_{F} n_{l}^{2} T_{F}^{2}\left\{\frac{1105}{81}+\frac{496}{27} \zeta_{3}-\frac{160}{27} \zeta_{2}+\left(-\frac{1585}{81}-\frac{112}{9} \zeta_{3}+\frac{160}{27} \zeta_{2}\right) L\right\} \\
& \left.+C_{F} n_{l}^{3} T_{F}^{3} \frac{32}{81}(1-L)\right]+\frac{1}{\varepsilon}\left[X_{V, 4}^{-1,0}+X_{V, 4}^{-1,1} L+N_{C}^{4}\left(-\frac{2073967}{11664}-\frac{441}{2} \zeta_{5}+\frac{178303}{648} \zeta_{3}-\frac{1}{3} \zeta_{3}^{2}+\frac{1141}{54} \zeta_{2}+\frac{118}{9} \zeta_{2} \zeta_{3}\right.\right. \\
& \left.-\frac{12142}{135} \zeta_{2}^{2}+\frac{1013}{45} \zeta_{2}^{3}\right) L+N_{C}^{3} n_{l} T_{F}\left(\frac{495349}{2916}+82 \zeta_{5}-\frac{2767}{18} \zeta_{3}+\frac{2531}{162} \zeta_{2}-\frac{32}{9} \zeta_{2} \zeta_{3}+\frac{694}{45} \zeta_{2}^{2}\right) L
\end{aligned}
$$




$$
\begin{aligned}
& +C_{F}^{4}\left\{\left(\frac{1972}{3}+16 c_{1}-516 \zeta_{5}-\frac{3244}{3} \zeta_{3}+\frac{2974}{3} \zeta_{2}+8 \zeta_{2} \zeta_{3}-504 \zeta_{2}^{2}-432 \ln (2) \zeta_{2}\right) L^{2}\right. \\
& +\left(-\frac{3025}{6}+\frac{4928}{9} \zeta_{3}-\frac{638}{3} \zeta_{2}+\frac{1232}{15} \zeta_{2}^{2}+96 \ln (2) \zeta_{2}\right) L^{3}+\left(\frac{1942}{9}-\frac{992}{9} \zeta_{3}+\frac{22}{3} \zeta_{2}\right) L^{4} \\
& \left.+\left(-62+6 \zeta_{2}\right) L^{5}+\frac{32}{3} L^{6}-\frac{10}{9} L^{7}\right\}+C_{A} C_{F}^{3}\left\{\left(-\frac{80599}{36}-8 c_{1}-74 \zeta_{5}+\frac{7045}{3} \zeta_{3}-\frac{15098}{27} \zeta_{2}-132 \zeta_{2} \zeta_{3}\right.\right. \\
& \left.+\frac{760}{3} \zeta_{2}^{2}+216 \ln (2) \zeta_{2}\right) L^{2}+\left(\frac{101510}{81}-\frac{5404}{9} \zeta_{3}+\frac{526}{9} \zeta_{2}-18 \zeta_{2}^{2}-48 \ln (2) \zeta_{2}\right) L^{3} \\
& \left.+\left(-\frac{18415}{54}+\frac{305}{3} \zeta_{3}+\frac{58}{9} \zeta_{2}\right) L^{4}+\left(\frac{161}{3}-9 \zeta_{2}\right) L^{5}-\frac{22}{9} L^{6}\right\}+C_{A}^{2} C_{F}^{2}\left\{\left(\frac{3887225}{2916}+260 \zeta_{5}-\frac{99733}{81} \zeta_{3}\right.\right. \\
& \left.+\frac{14699}{81} \zeta_{2}+\frac{328}{3} \zeta_{2} \zeta_{3}+\frac{1232}{45} \zeta_{2}^{2}\right) L^{2}+\left(-\frac{190591}{486}+\frac{2024}{27} \zeta_{3}+\frac{4829}{54} \zeta_{2}-\frac{412}{15} \zeta_{2}^{2}\right) L^{3}+\left(\frac{38237}{972}-\frac{154}{27} \zeta_{2}\right) L^{4} \\
& \left.-\frac{605}{324} L^{5}\right\}+C_{F}^{3} n_{l} T_{F}\left\{\left(\frac{9925}{27}-\frac{2560}{9} \zeta_{3}+\frac{4120}{27} \zeta_{2}+\frac{104}{15} \zeta_{2}^{2}\right) L^{2}+\left(-\frac{26932}{81}-\frac{64}{9} \zeta_{3}-\frac{428}{9} \zeta_{2}\right) L^{3}\right. \\
& \left.+\left(\frac{2902}{27}+\frac{100}{9} \zeta_{2}\right) L^{4}-\frac{52}{3} L^{5}+\frac{8}{9} L^{6}\right\}+C_{A} C_{F}^{2} n_{l} T_{F}\left\{\left(-\frac{519812}{729}+\frac{17068}{81} \zeta_{3}-\frac{1912}{9} \zeta_{2}-\frac{208}{45} \zeta_{2}^{2}\right) L^{2}\right. \\
& \left.+\left(\frac{55448}{243}+\frac{416}{27} \zeta_{3}-\frac{400}{27} \zeta_{2}\right) L^{3}+\left(-\frac{6446}{243}+\frac{56}{27} \zeta_{2}\right) L^{4}+\frac{110}{81} L^{5}\right\}+C_{F}^{2} n_{l}^{2} T_{F}^{2}\left\{\left(-\frac{47536}{729}-\frac{1184}{81} \zeta_{3}\right.\right. \\
& \left.\left.-\frac{1720}{27} \zeta_{2}+\frac{3424}{135} \zeta_{2}^{2}\right) L+\left(\frac{52316}{729}+\frac{512}{81} \zeta_{3}+\frac{3568}{81} \zeta_{2}\right) L^{2}+\left(-\frac{6536}{243}-\frac{104}{27} \zeta_{2}\right) L^{3}+\frac{980}{243} L^{4}-\frac{20}{81} L^{5}\right\} \\
& \left.+C_{A} C_{F} n_{l}^{2} T_{F}^{2}\left\{\left(\frac{923}{162}+\frac{1120}{27} \zeta_{3}-\frac{304}{81} \zeta_{2}-\frac{112}{15} \zeta_{2}^{2}\right) L\right\}+C_{F} n_{l}^{3} T_{F}^{3}\left\{\left(-\frac{32}{81}+\frac{64}{27} \zeta_{3}\right) L\right\}\right] \\
& +\left[X_{V, 4}^{0,0}+X_{V, 4}^{0,1} L+X_{V, 4}^{0,2} L^{2}+N_{C}^{4}\left(\frac{201483937}{279936}-\frac{149}{3} \zeta_{5}-\frac{308201}{486} \zeta_{3}+\frac{1193}{12} \zeta_{3}^{2}+\frac{2972341}{7776} \zeta_{2}+\frac{839}{108} \zeta_{2} \zeta_{3}\right.\right. \\
& \left.-\frac{3451}{80} \zeta_{2}^{2}+\frac{761}{36} \zeta_{2}^{3}\right) L^{2}+N_{C}^{3} n_{l} T_{F}\left(-\frac{2276657}{34992}+\frac{314}{3} \zeta_{5}+\frac{7945}{243} \zeta_{3}-\frac{93347}{486} \zeta_{2}-\frac{55}{27} \zeta_{2} \zeta_{3}-\frac{12979}{540} \zeta_{2}^{2}\right) L^{2} \\
& +C_{F}^{4}\left\{\left(-\frac{16252}{9}-16 c_{1}+1060 \zeta_{5}+\frac{27328}{9} \zeta_{3}-\frac{16273}{9} \zeta_{2}-\frac{1640}{9} \zeta_{2} \zeta_{3}+\frac{9596}{15} \zeta_{2}^{2}+368 \ln (2) \zeta_{2}\right) L^{3}\right. \\
& +\left(\frac{20759}{24}-822 \zeta_{3}+\frac{571}{2} \zeta_{2}-\frac{299}{3} \zeta_{2}^{2}-56 \ln (2) \zeta_{2}\right) L^{4}+\left(-\frac{47299}{180}+\frac{1144}{9} \zeta_{3}-\frac{38}{3} \zeta_{2}\right) L^{5}+\left(\frac{2518}{45}-\frac{44}{9} \zeta_{2}\right) L^{6} \\
& \left.-\frac{343}{45} L^{7}+\frac{27}{40} L^{8}\right\}+C_{A} C_{F}^{3}\left\{\left(\frac{3658325}{486}+8 c_{1}-370 \zeta_{5}-\frac{188444}{27} \zeta_{3}+\frac{60428}{27} \zeta_{2}+400 \zeta_{2} \zeta_{3}-\frac{60979}{90} \zeta_{2}^{2}\right.\right. \\
& \left.-\frac{728}{3} \ln (2) \zeta_{2}\right) L^{3}+\left(-\frac{1588517}{648}+\frac{10637}{9} \zeta_{3}-\frac{4825}{27} \zeta_{2}+\frac{1531}{30} \zeta_{2}^{2}+28 \ln (2) \zeta_{2}\right) L^{4}+\left(\frac{176119}{360}-116 \zeta_{3}-13 \zeta_{2}\right) L^{5} \\
& \left.+\left(-\frac{33517}{540}+\frac{22}{3} \zeta_{2}\right) L^{6}+\frac{209}{60} L^{7}\right\}+C_{A}^{2} C_{F}^{2}\left\{\left(-\frac{4578454}{729}-540 \zeta_{5}+\frac{38435}{9} \zeta_{3}-\frac{299959}{324} \zeta_{2}-\frac{1976}{9} \zeta_{2} \zeta_{3}+\frac{6752}{45} \zeta_{2}^{2}\right.\right. \\
& \left.\left.+\frac{88}{3} \ln (2) \zeta_{2}\right) L^{3}+\left(\frac{1220761}{972}-\frac{6413}{18} \zeta_{3}-\frac{691}{9} \zeta_{2}+\frac{1486}{45} \zeta_{2}^{2}\right) L^{4}-\left(\frac{1363133}{9720}-\frac{1001}{54} \zeta_{2}\right) L^{5}+\frac{3751}{540} L^{6}\right\} \\
& +C_{A}^{3} C_{F}\left\{\left(\frac{467107}{486}-\frac{968}{3} \zeta_{3}-\frac{694}{27} \zeta_{2}+\frac{484}{15} \zeta_{2}^{2}\right) L^{3}+\left(-\frac{33803}{324}+\frac{121}{9} \zeta_{2}\right) L^{4}+\frac{1331}{270} L^{5}\right\} \\
& +C_{F}^{3} n_{l} T_{F}\left\{\left(-\frac{427058}{243}+\frac{30664}{27} \zeta_{3}-\frac{19252}{27} \zeta_{2}+\frac{290}{9} \zeta_{2}^{2}+\frac{64}{3} \ln (2) \zeta_{2}\right) L^{3}\right. \\
& \left.+\left(\frac{115585}{162}-\frac{896}{9} \zeta_{3}+\frac{2660}{27} \zeta_{2}\right) L^{4}+\left(-\frac{14269}{90}-8 \zeta_{2}\right) L^{5}+\frac{2807}{135} L^{6}-\frac{19}{15} L^{7}\right\}
\end{aligned}
$$




$$
\begin{aligned}
& +C_{A} C_{F}^{2} n_{l} T_{F}\left\{\left(\frac{2759932}{729}-\frac{98272}{81} \zeta_{3}+\frac{64054}{81} \zeta_{2}-\frac{160}{3} \zeta_{2}^{2}-\frac{32}{3} \ln (2) \zeta_{2}\right) L^{3}+\left(-\frac{21805}{27}+78 \zeta_{3}-\frac{1354}{81} \zeta_{2}\right) L^{4}\right. \\
& \left.+\left(\frac{116653}{1215}-\frac{182}{27} \zeta_{2}\right) L^{5}-\frac{682}{135} L^{6}\right\}+C_{A}^{2} C_{F} n_{l} T_{F}\left\{\left(-\frac{25553}{27}+176 \zeta_{3}-\frac{712}{27} \zeta_{2}-\frac{176}{15} \zeta_{2}^{2}\right) L^{3}\right. \\
& \left.+\left(\frac{2920}{27}-\frac{88}{9} \zeta_{2}\right) L^{4}-\frac{242}{45} L^{5}\right\}+C_{F}^{2} n_{l}^{2} T_{F}^{2}\left\{\left(\frac{2471390}{2187}-\frac{60640}{243} \zeta_{3}+\frac{54284}{81} \zeta_{2}+\frac{3596}{135} \zeta_{2}^{2}\right) L^{2}\right. \\
& \left.+\left(-\frac{370744}{729}+\frac{1616}{81} \zeta_{3}-\frac{12172}{81} \zeta_{2}\right) L^{3}+\left(\frac{28904}{243}+\frac{1064}{81} \zeta_{2}\right) L^{4}-\frac{18826}{1215} L^{5}+\frac{124}{135} L^{6}\right\} \\
& +C_{A} C_{F} n_{l}^{2} T_{F}^{2}\left\{\left(\frac{8996449}{2916}-\frac{2464}{9} \zeta_{5}-\frac{86416}{81} \zeta_{3}+\frac{459772}{243} \zeta_{2}+\frac{608}{9} \zeta_{2} \zeta_{3}+\frac{1232}{15} \zeta_{2}^{2}\right) L\right. \\
& \left.+\left(-\frac{308686}{243}+\frac{2032}{9} \zeta_{3}-\frac{11440}{27} \zeta_{2}+\frac{80}{3} \zeta_{2}^{2}\right) L^{2}+\left(\frac{23302}{81}-\frac{64}{3} \zeta_{3}+\frac{736}{27} \zeta_{2}\right) L^{3}+\left(-\frac{968}{27}+\frac{16}{9} \zeta_{2}\right) L^{4}+\frac{88}{45} L^{5}\right\} \\
& +C_{F} n_{l}^{3} T_{F}^{3}\left\{\left(-\frac{558992}{2187}-\frac{320}{81} \zeta_{3}-\frac{12992}{81} \zeta_{2}-\frac{832}{45} \zeta_{2}^{2}\right) L+\left(\frac{79280}{729}+\frac{1216}{27} \zeta_{2}\right) L^{2}\right. \\
& \left.\left.+\left(-\frac{6496}{243}-\frac{128}{27} \zeta_{2}\right) L^{3}+\frac{304}{81} L^{4}-\frac{32}{135} L^{5}\right\}\right] \\
& F_{S}^{4}=\frac{1}{\varepsilon^{4}}\left[C_{F}^{4} \frac{2}{3}(1-L)^{4}+C_{A} C_{F}^{3} \frac{22}{3}(1-L)^{3}+C_{A}^{2} C_{F}^{2} \frac{1331}{54}(1-L)^{2}+C_{A}^{3} C_{F} \frac{1331}{54}(1-L)-C_{F}^{3} n_{l} T_{F} \frac{8}{3}(1-L)^{3}\right. \\
& -C_{A} C_{F}^{2} n_{l} T_{F} \frac{484}{27}(1-L)^{2}-C_{A}^{2} C_{F} n_{l} T_{F} \frac{242}{9}(1-L)+C_{F}^{2} n_{l}^{2} T_{F}^{2} \frac{88}{27}(1-L)^{2}+C_{A} C_{F} n_{l}^{2} T_{F}^{2} \frac{88}{9}(1-L) \\
& \left.-C_{F} n_{l}^{3} T_{F}^{3} \frac{32}{27}(1-L)\right]+\frac{1}{\varepsilon^{3}}\left[C_{F}^{4}\left\{\frac{8}{3}-\frac{8}{3} \zeta_{2}-8\left(1-\zeta_{2}\right) L+\left(\frac{28}{3}-8 \zeta_{2}\right) L^{2}+\left(-\frac{20}{3}+\frac{8}{3} \zeta_{2}\right) L^{3}+4 L^{4}-\frac{4}{3} L^{5}\right\}\right. \\
& +C_{F}^{3} C_{A}\left\{\frac{34}{9}-4 \zeta_{3}-\frac{32}{3} \zeta_{2}+\left(\frac{22}{3}+8 \zeta_{3}+\frac{52}{3} \zeta_{2}\right) L-\left(\frac{56}{3}+4 \zeta_{3}+\frac{8}{3} \zeta_{2}\right) L^{2}+\left(\frac{2}{9}-4 \zeta_{2}\right) L^{3}+\frac{22}{3} L^{4}\right\} \\
& +C_{F}^{2} C_{A}^{2}\left\{-\frac{3545}{81}-\frac{154}{9} \zeta_{3}-\frac{22}{27} \zeta_{2}+\left(\frac{9928}{81}+\frac{154}{9} \zeta_{3}-\frac{440}{27} \zeta_{2}\right) L+\left(-\frac{5657}{81}+\frac{154}{9} \zeta_{2}\right) L^{2}-\frac{242}{27} L^{3}\right\} \\
& +C_{F} C_{A}^{3}\left\{-\frac{12661}{162}-\frac{121}{9} \zeta_{3}+\frac{121}{9} \zeta_{2}+\left(\frac{14839}{162}-\frac{121}{9} \zeta_{2}\right) L\right\}+C_{F}^{3} n_{l} T_{F}\left\{\frac{40}{9}+\frac{16}{3} \zeta_{2}+\left(-\frac{40}{3}-\frac{32}{3} \zeta_{2}\right) L\right. \\
& \left.+\left(\frac{32}{3}+\frac{16}{3} \zeta_{2}\right) L^{2}+\frac{8}{9} L^{3}-\frac{8}{3} L^{4}\right\}+C_{F}^{2} C_{A} n_{l} T_{F}\left\{\frac{3764}{81}+\frac{56}{9} \zeta_{3}+\frac{184}{27} \zeta_{2}+\left(-\frac{7900}{81}-\frac{56}{9} \zeta_{3}-\frac{16}{27} \zeta_{2}\right) L\right. \\
& \left.+\left(\frac{3608}{81}-\frac{56}{9} \zeta_{2}\right) L^{2}+\frac{176}{27} L^{3}\right\}+C_{F} C_{A}^{2} n_{l} T_{F}\left\{\frac{730}{9}+\frac{88}{9} \zeta_{3}-\frac{88}{9} \zeta_{2}+\left(-\frac{818}{9}+\frac{88}{9} \zeta_{2}\right) L\right\} \\
& +C_{F}^{2} n_{l}^{2} T_{F}^{2}\left\{-\frac{800}{81}-\frac{64}{27} \zeta_{2}+\left(\frac{1360}{81}+\frac{64}{27} \zeta_{2}\right) L-\frac{464}{81} L^{2}-\frac{32}{27} L^{3}\right\}+C_{F} C_{A} n_{l}^{2} T_{F}^{2}\left\{-\frac{664}{27}-\frac{16}{9} \zeta_{3}+\frac{16}{9} \zeta_{2}\right. \\
& \left.\left.+\left(\frac{712}{27}-\frac{16}{9} \zeta_{2}\right) L\right\}+C_{F} n_{l}^{3} T_{F}^{3}\left\{\frac{160}{81}-\frac{160}{81} L\right\}\right]+\frac{1}{\varepsilon^{2}}\left[C _ { F } ^ { 4 } \left\{\frac{142}{3}-\frac{304}{3} \zeta_{3}+\frac{28}{3} \zeta_{2}-\frac{236}{5} \zeta_{2}^{2}\right.\right. \\
& +\left(-\frac{308}{3}+256 \zeta_{3}-\frac{128}{3} \zeta_{2}+\frac{472}{5} \zeta_{2}^{2}\right) L+\left(70-208 \zeta_{3}+52 \zeta_{2}-\frac{236}{5} \zeta_{2}^{2}\right) L^{2}+\left(-\frac{196}{9}+\frac{160}{3} \zeta_{3}-\frac{40}{3} \zeta_{2}\right) L^{3} \\
& \left.+\left(9-\frac{16}{3} \zeta_{2}\right) L^{4}-\frac{10}{3} L^{5}+\frac{13}{9} L^{6}\right\}+C_{F}^{3} C_{A}\left\{\frac{545}{27}-148 \zeta_{3}+\frac{830}{9} \zeta_{2}+8 \zeta_{2} \zeta_{3}-\frac{1436}{15} \zeta_{2}^{2}\right. \\
& +\left(\frac{431}{9}+148 \zeta_{3}-\frac{1798}{9} \zeta_{2}-8 \zeta_{2} \zeta_{3}+\frac{1574}{15} \zeta_{2}^{2}\right) L+\left(-\frac{808}{9}+48 \zeta_{3}+\frac{776}{9} \zeta_{2}-\frac{46}{5} \zeta_{2}^{2}\right) L^{2} \\
& \left.+\left(\frac{1390}{27}-48 \zeta_{3}+\frac{40}{3} \zeta_{2}\right) L^{3}+\left(-\frac{503}{18}+8 \zeta_{2}\right) L^{4}-\frac{11}{6} L^{5}\right\}
\end{aligned}
$$




$$
\begin{aligned}
& +C_{F}^{2} C_{A}^{2}\left\{-\frac{13553}{162}-24 \zeta_{5}+\frac{3776}{27} \zeta_{3}+2 \zeta_{3}^{2}+\frac{6374}{81} \zeta_{2}+\frac{100}{9} \zeta_{2} \zeta_{3}+\frac{79}{45} \zeta_{2}^{2}+\left(\frac{254}{9}+24 \zeta_{5}-\frac{6986}{27} \zeta_{3}+\frac{160}{81} \zeta_{2}\right.\right. \\
& \left.\left.-\frac{4}{3} \zeta_{2} \zeta_{3}-\frac{697}{45} \zeta_{2}^{2}\right) L+\left(\frac{343}{18}+\frac{902}{9} \zeta_{3}-\frac{704}{9} \zeta_{2}+\frac{206}{15} \zeta_{2}^{2}\right) L^{2}+\left(\frac{4418}{81}-\frac{110}{9} \zeta_{2}\right) L^{3}-\frac{121}{81} L^{4}\right\} \\
& +C_{F} C_{A}^{3}\left\{\frac{4981}{54}-33 \zeta_{5}+\frac{2341}{27} \zeta_{3}-\frac{1978}{27} \zeta_{2}+\frac{22}{3} \zeta_{2} \zeta_{3}+\frac{242}{15} \zeta_{2}^{2}+\left(-\frac{287}{2}-\frac{121}{9} \zeta_{3}+\frac{1780}{27} \zeta_{2}-\frac{242}{15} \zeta_{2}^{2} L\right\}\right. \\
& +C_{F}^{3} n_{l} T_{F}\left\{-\frac{901}{27}+\frac{256}{3} \zeta_{3}-\frac{184}{9} \zeta_{2}+\frac{472}{15} \zeta_{2}^{2}+\left(\frac{379}{9}-\frac{416}{3} \zeta_{3}+\frac{344}{9} \zeta_{2}-\frac{472}{15} \zeta_{2}^{2}\right) L+\left(\frac{28}{9}+\frac{160}{3} \zeta_{3}-\frac{64}{9} \zeta_{2}\right) L^{2}\right. \\
& \left.+\left(-\frac{560}{27}-\frac{32}{3} \zeta_{2}\right) L^{3}+\frac{74}{9} L^{4}+\frac{2}{3} L^{5}\right\}+C_{F}^{2} C_{A} n_{l} T_{F}\left\{-\frac{35}{9}-\frac{148}{3} \zeta_{3}-\frac{3476}{81} \zeta_{2}-\frac{32}{9} \zeta_{2} \zeta_{3}+\frac{196}{45} \zeta_{2}^{2}\right. \\
& \left.+\left(\frac{2219}{81}+\frac{976}{9} \zeta_{3}+\frac{332}{81} \zeta_{2}-\frac{196}{45} \zeta_{2}^{2}\right) L+\left(\frac{788}{81}-\frac{520}{9} \zeta_{3}+\frac{1024}{27} \zeta_{2}\right) L^{2}+\left(-\frac{2888}{81}+\frac{40}{9} \zeta_{2}\right) L^{3}+\frac{88}{81} L^{4}\right\} \\
& +C_{F} C_{A}^{2} n_{l} T_{F}\left\{-\frac{13241}{162}+12 \zeta_{5}-\frac{2284}{27} \zeta_{3}+\frac{1228}{27} \zeta_{2}-\frac{8}{3} \zeta_{2} \zeta_{3}-\frac{88}{15} \zeta_{2}^{2}+\left(\frac{19313}{162}+\frac{352}{9} \zeta_{3}-\frac{1156}{27} \zeta_{2}+\frac{88}{15} \zeta_{2}^{2}\right) L\right\} \\
& +C_{F}^{2} n_{l}^{2} T_{F}^{2}\left\{\frac{964}{81}-\frac{224}{27} \zeta_{3}+\frac{320}{81} \zeta_{2}-\left(\frac{844}{81}-\frac{224}{27} \zeta_{3}-\frac{64}{81} \zeta_{2}\right) L+\left(-\frac{520}{81}-\frac{128}{27} \zeta_{2}\right) L^{2}+\frac{416}{81} L^{3}-\frac{16}{81} L^{4}\right\} \\
& \left.+C_{F} C_{A} n_{l}^{2} T_{F}^{2}\left\{\frac{1105}{81}+\frac{496}{27} \zeta_{3}-\frac{160}{27} \zeta_{2}+\left(-\frac{1585}{81}-\frac{112}{9} \zeta_{3}+\frac{160}{27} \zeta_{2}\right) L\right\}+C_{F} n_{l}^{3} T_{F}^{3}\left\{\frac{32}{81}-\frac{32}{81} L\right\}\right] \\
& +\frac{1}{\varepsilon}\left[X_{S, 4}^{-1,0}+X_{S, 4}^{-1,1} L+N_{C}^{4}\left(\frac{598439}{11664}-\frac{429}{2} \zeta_{5}+\frac{42851}{324} \zeta_{3}-\frac{1}{3} \zeta_{3}^{2}+\frac{1327}{108} \zeta_{2}+\frac{487}{9} \zeta_{2} \zeta_{3}-\frac{40243}{540} \zeta_{2}^{2}+\frac{1013}{45} \zeta_{2}^{3}\right) L\right. \\
& +N_{C}^{3} n_{l} T_{F}\left(-\frac{117913}{5832}+82 \zeta_{5}-\frac{2021}{18} \zeta_{3}+\frac{6017}{162} \zeta_{2}-\frac{32}{9} \zeta_{2} \zeta_{3}-\frac{317}{45} \zeta_{2}^{2}\right) L+C_{F}^{4}\left\{\left(-\frac{293}{3}-516 \zeta_{5}-\frac{2068}{3} \zeta_{3}\right.\right. \\
& \left.+\frac{424}{3} \zeta_{2}+8 \zeta_{2} \zeta_{3}-168 \zeta_{2}^{2}+528 \ln (2) \zeta_{2}\right) L^{2}+\left(-118+\frac{3560}{9} \zeta_{3}-\frac{388}{3} \zeta_{2}+\frac{1232}{15} \zeta_{2}^{2}\right) L^{3} \\
& \left.+\left(\frac{271}{9}-\frac{992}{9} \zeta_{3}+\frac{118}{3} \zeta_{2}\right) L^{4}+\left(-\frac{29}{3}+6 \zeta_{2}\right) L^{5}+2 L^{6}-\frac{10}{9} L^{7}\right\}-C_{F}^{3} C_{A}\left\{\left(\frac{5315}{27}+74 \zeta_{5}-\frac{5002}{3} \zeta_{3}+\frac{8681}{27} \zeta_{2}\right.\right. \\
& \left.+132 \zeta_{2} \zeta_{3}-\frac{2342}{15} \zeta_{2}^{2}+264 \ln (2) \zeta_{2}\right) L^{2}-\left(\frac{16982}{81}-\frac{3244}{9} \zeta_{3}-\frac{140}{9} \zeta_{2}-18 \zeta_{2}^{2}\right) L^{3}+\left(\frac{4225}{54}-\frac{305}{3} \zeta_{3}+\frac{158}{9} \zeta_{2}\right) L^{4} \\
& \left.-\left(\frac{223}{6}-9 \zeta_{2}\right) L^{5}+\frac{22}{9} L^{6}\right\}+C_{F}^{2} C_{A}^{2}\left\{\left(\frac{96527}{729}+260 \zeta_{5}-\frac{90526}{81} \zeta_{3}+\frac{30721}{162} \zeta_{2}+\frac{328}{3} \zeta_{2} \zeta_{3}+\frac{88}{9} \zeta_{2}^{2}\right) L^{2}\right. \\
& \left.+\left(-\frac{53009}{243}+\frac{2024}{27} \zeta_{3}+\frac{4631}{54} \zeta_{2}-\frac{412}{15} \zeta_{2}^{2}\right) L^{3}+\left(\frac{27347}{972}-\frac{154}{27} \zeta_{2}\right) L^{4}-\frac{605}{324} L^{5}\right\} \\
& +C_{F}^{3} n_{l} T_{F}\left\{\left(-\frac{1018}{27}-\frac{3280}{9} \zeta_{3}+\frac{4084}{27} \zeta_{2}+\frac{104}{15} \zeta_{2}^{2}\right) L^{2}+\left(-\frac{2272}{81}-\frac{64}{9} \zeta_{3}-\frac{176}{9} \zeta_{2}\right) L^{3}\right. \\
& \left.+\left(\frac{754}{27}+\frac{100}{9} \zeta_{2}\right) L^{4}-\frac{34}{3} L^{5}+\frac{8}{9} L^{6}\right\}+C_{F}^{2} C_{A} n_{l} T_{F}\left\{\left(-\frac{59948}{729}+\frac{24088}{81} \zeta_{3}-\frac{524}{3} \zeta_{2}-\frac{208}{45} \zeta_{2}^{2}\right) L^{2}\right. \\
& \left.+\left(\frac{27944}{243}+\frac{416}{27} \zeta_{3}-\frac{364}{27} \zeta_{2}\right) L^{3}+\left(-\frac{4466}{243}+\frac{56}{27} \zeta_{2}\right) L^{4}+\frac{110}{81} L^{5}\right\} \\
& +C_{F}^{2} n_{l}^{2} T_{F}^{2}\left\{\left(\frac{18740}{729}-\frac{2624}{81} \zeta_{3}-\frac{1840}{27} \zeta_{2}+\frac{3424}{135} \zeta_{2}^{2}\right) L+\left(\frac{5984}{729}+\frac{512}{81} \zeta_{3}+\frac{2632}{81} \zeta_{2}\right) L^{2}\right. \\
& \left.+\left(-\frac{2576}{243}-\frac{104}{27} \zeta_{2}\right) L^{3}+\frac{620}{243} L^{4}-\frac{20}{81} L^{5}\right\}+C_{F} C_{A} n_{l}^{2} T_{F}^{2}\left\{\left(\frac{923}{162}+\frac{1120}{27} \zeta_{3}-\frac{304}{81} \zeta_{2}-\frac{112}{15} \zeta_{2}^{2}\right) L\right\}
\end{aligned}
$$




$$
\begin{aligned}
& \left.+C_{F} n_{l}^{3} T_{F}^{3}\left\{\left(-\frac{32}{81}+\frac{64}{27} \zeta_{3}\right) L\right\}\right]+\left[X_{S, 4}^{0,0}+X_{S, 4}^{0,1} L+X_{S, 4}^{0,2} L^{2}+N_{C}^{4}\left\{\left(-\frac{31524563}{69984}-\frac{455}{3} \zeta_{5}+\frac{194209}{1944} \zeta_{3}\right.\right.\right. \\
& \left.\left.+\frac{1193}{12} \zeta_{3}^{2}+\frac{26833}{486} \zeta_{2}-\frac{187}{108} \zeta_{2} \zeta_{3}-\frac{7343}{120} \zeta_{2}^{2}+\frac{761}{36} \zeta_{2}^{3}\right) L^{2}\right\}+N_{C}^{3} n_{l} T_{F}\left\{\left(\frac{472810}{2187}+\frac{314}{3} \zeta_{5}-\frac{23231}{243} \zeta_{3}+\frac{23}{243} \zeta_{2}\right.\right. \\
& \left.\left.-\frac{55}{27} \zeta_{2} \zeta_{3}-\frac{271}{540} \zeta_{2}^{2}\right) L^{2}\right\}+C_{F}^{4}\left\{\left(\frac{239}{9}+1060 \zeta_{5}+\frac{11132}{9} \zeta_{3}-\frac{3964}{9} \zeta_{2}-\frac{1640}{9} \zeta_{2} \zeta_{3}+\frac{2852}{15} \zeta_{2}^{2}-528 \ln (2) \zeta_{2}\right) L^{3}\right. \\
& \left.+\left(\frac{275}{2}-\frac{1388}{3} \zeta_{3}+171 \zeta_{2}-\frac{299}{3} \zeta_{2}^{2}\right) L^{4}+\left(-\frac{1321}{45}+\frac{1144}{9} \zeta_{3}-\frac{146}{3} \zeta_{2}\right) L^{5}+\left(\frac{731}{90}-\frac{44}{9} \zeta_{2}\right) L^{6}-\frac{43}{45} L^{7}+\frac{27}{40} L^{8}\right\} \\
& +C_{F}^{3} C_{A}\left\{\left(\frac{133270}{243}-370 \zeta_{5}-\frac{116066}{27} \zeta_{3}+\frac{36173}{27} \zeta_{2}+400 \zeta_{2} \zeta_{3}-\frac{40567}{90} \zeta_{2}^{2}+264 \ln (2) \zeta_{2}\right) L^{3}\right. \\
& \left.+\left(-\frac{49319}{162}+\frac{6470}{9} \zeta_{3}-\frac{3502}{27} \zeta_{2}+\frac{1531}{30} \zeta_{2}^{2}\right) L^{4}+\left(\frac{7771}{90}-116 \zeta_{3}+14 \zeta_{2}\right) L^{5}+\left(-\frac{16687}{540}+\frac{22}{3} \zeta_{2}\right) L^{6}+\frac{209}{60} L^{7}\right\} \\
& +C_{F}^{2} C_{A}^{2}\left\{\left(-\frac{411373}{729}-540 \zeta_{5}+\frac{28843}{9} \zeta_{3}-\frac{52132}{81} \zeta_{2}-\frac{1976}{9} \zeta_{2} \zeta_{3}+\frac{8336}{45} \zeta_{2}^{2}\right) L^{3}\right. \\
& \left.+\left(\frac{376009}{972}-\frac{6413}{18} \zeta_{3}-\frac{383}{9} \zeta_{2}+\frac{1486}{45} \zeta_{2}^{2}\right) L^{4}+\left(-\frac{203297}{2430}+\frac{1001}{54} \zeta_{2}\right) L^{5}+\frac{3751}{540} L^{6}\right\} \\
& +C_{F} C_{A}^{3}\left\{\left(\frac{83618}{243}-\frac{968}{3} \zeta_{3}-\frac{694}{27} \zeta_{2}+\frac{484}{15} \zeta_{2}^{2}\right) L^{3}+\left(-\frac{5456}{81}+\frac{121}{9} \zeta_{2}\right) L^{4}+\frac{1331}{270} L^{5}\right\} \\
& +C_{F}^{3} n_{l} T_{F}\left\{\left(-\frac{13454}{243}+\frac{30448}{27} \zeta_{3}-\frac{11980}{27} \zeta_{2}+\frac{290}{9} \zeta_{2}^{2}\right) L^{3}+\left(\frac{5243}{81}-\frac{896}{9} \zeta_{3}+\frac{1724}{27} \zeta_{2}\right) L^{4}\right. \\
& \left.+\left(-\frac{1352}{45}-8 \zeta_{2}\right) L^{5}+\frac{1277}{135} L^{6}-\frac{19}{15} L^{7}\right\}+C_{F}^{2} C_{A} n_{l} T_{F}\left\{\left(\frac{269308}{729}-\frac{87616}{81} \zeta_{3}+\frac{40528}{81} \zeta_{2}-\frac{160}{3} \zeta_{2}^{2}\right) L^{3}\right. \\
& \left.+\left(-\frac{18701}{81}+78 \zeta_{3}-\frac{2362}{81} \zeta_{2}\right) L^{4}+\left(\frac{66658}{1215}-\frac{182}{27} \zeta_{2}\right) L^{5}-\frac{682}{135} L^{6}\right\}+C_{F} C_{A}^{2} n_{l} T_{F}\left\{\left(-\frac{8693}{27}+176 \zeta_{3}-\frac{712}{27} \zeta_{2}\right.\right. \\
& \left.\left.-\frac{176}{15} \zeta_{2}^{2}\right) L^{3}+\left(\frac{1831}{27}-\frac{88}{9} \zeta_{2}\right) L^{4}-\frac{242}{45} L^{5}\right\}+C_{F}^{2} n_{l}^{2} T_{F}^{2}\left\{\left(-\frac{47206}{2187}-\frac{78928}{243} \zeta_{3}+\frac{22760}{81} \zeta_{2}+\frac{3596}{135} \zeta_{2}^{2}\right) L^{2}\right. \\
& \left.+\left(-\frac{37744}{729}+\frac{1616}{81} \zeta_{3}-\frac{7168}{81} \zeta_{2}\right) L^{3}+\left(\frac{7256}{243}+\frac{1064}{81} \zeta_{2}\right) L^{4}-\frac{9736}{1215} L^{5}+\frac{124}{135} L^{6}\right\} \\
& +C_{F} C_{A} n_{l}^{2} T_{F}^{2}\left\{\left(-\frac{823055}{2916}+96 \zeta_{4}-\frac{2464}{9} \zeta_{5}-\frac{68272}{81} \zeta_{3}+\frac{146572}{243} \zeta_{2}+\frac{608}{9} \zeta_{2} \zeta_{3}+\frac{160}{3} \zeta_{2}^{2}\right) L\right. \\
& \left.+\left(-\frac{26293}{243}+\frac{2032}{9} \zeta_{3}-\frac{6400}{27} \zeta_{2}+\frac{80}{3} \zeta_{2}^{2}\right) L^{2}+\left(\frac{7030}{81}-\frac{64}{3} \zeta_{3}+\frac{736}{27} \zeta_{2}\right) L^{3}+\left(-\frac{572}{27}+\frac{16}{9} \zeta_{2}\right) L^{4}+\frac{88}{45} L^{5}\right\} \\
& +C_{F} n_{l}^{3} T_{F}^{3}\left\{\left(-\frac{20864}{2187}-\frac{320}{81} \zeta_{3}-\frac{3200}{81} \zeta_{2}-\frac{832}{45} \zeta_{2}^{2}\right) L+\left(\frac{8000}{729}+\frac{640}{27} \zeta_{2}\right) L^{2}\right. \\
& \left.\left.+\left(-\frac{1600}{243}-\frac{128}{27} \zeta_{2}\right) L^{3}+\frac{160}{81} L^{4}-\frac{32}{135} L^{5}\right\}\right] \text {. }
\end{aligned}
$$

To obtain $X_{I, 4}^{-1,0}$ for $I=V, S$ one needs the massive quark anomalous dimension at four-loop order, $\gamma_{Q}^{(3)}$, and the complete contributions from three-loop order are necessary as well. On the other hand, $X_{I, 4}^{-1,1}$ lacks the nonplanar contributions only, which can be obtained once the massless cusp anomalous dimension $A_{q}^{(4)}$ is known completely. It similarly follows for the remaining of the functions $X_{I, 4}^{0, k}$.
The exact result for the color-planar and complete light quark contributions for the vector and scalar form factors are available [31-34] now. We successfully cross-checked our results in the corresponding limit. An interesting point to note is that similar to the coefficients $g_{I}^{n, k}$, the coefficients $\mathcal{C}_{I}^{n, k}$ are also in accordance with principle of leading transcendentality, i.e., the leading transcendental terms for each order in the $\varepsilon$ expansion are the same in both 
the vector and scalar cases. This aspect is of importance considering form factors in supersymmetric $\mathcal{N}=4 \mathrm{SYM}$ theories.

Finally, we would like to mention that the $Q^{2}$-dependent parts of $\hat{F}_{I}$ and the massless form factor $\bar{F}_{I}=F_{I}(m=0)$ are the same. The universal function $Z_{I}^{(m \mid 0)}$ [10] is then given by

$$
\begin{aligned}
& Z_{I}^{(m \mid 0)}\left(\frac{m^{2}}{\mu^{2}}, a_{s}, \varepsilon\right) \\
& =F_{I}\left(\frac{Q^{2}}{\mu^{2}}, \frac{m^{2}}{\mu^{2}}, a_{s}, \varepsilon\right)\left(\bar{F}_{I}\left(\frac{Q^{2}}{\mu^{2}}, a_{s}, \varepsilon\right)\right)^{-1} \\
& \quad=\mathcal{C}_{I}\left(a_{s}, \varepsilon\right) \hat{F}_{I}\left(\frac{Q^{2}}{\mu^{2}}, \frac{m^{2}}{\mu^{2}}, a_{s}, \varepsilon\right)\left(\bar{F}_{I}\left(\frac{Q^{2}}{\mu^{2}}, a_{s}, \varepsilon\right)\right)^{-1} .
\end{aligned}
$$

\section{CONCLUSION}

We presented a systematic study of the massive form factors at $Q^{2} \gg m^{2}$ at three-loop order by solving the associated evolution equations both in the vector and scalar cases. The universal structure of the IR singularities, along with the interplay of the various anomalous dimensions, has enabled us to obtain all asymptotic corrections at threeloop order for all logarithmic contributions. We also obtained partial four-loop results, still containing pieces, which can only be determined by performing a four-loop calculation. The Dirac axial-vector and vector form factor, and likewise the pseudoscalar and scalar form factors, agree in this limit, while the Pauli vector form factors vanish. We remark that there are additional corrections due to massive internal quark loops, which have not been considered in the present paper and are the subject of further investigations. The present results constrain future calculations and may serve as important checks.

\section{ACKNOWLEDGMENTS}

We would like to thank J. Ablinger, T. Ahmed, S. Moch, V. Ravindran, and C. Schneider for discussions and J. C. Collins for a comment. This work has been funded in part by EU TMR network SAGEX agreement No. 764850 (Marie Skłodowska-Curie) and COST action CA16201: Unraveling new physics at the LHC through the precision frontier.
[1] S. Catani, The singular behavior of QCD amplitudes at two loop order, Phys. Lett. B 427, 161 (1998).

[2] G. F. Sterman and M. E. Tejeda-Yeomans, Multiloop amplitudes and resummation, Phys. Lett. B 552, 48 (2003).

[3] T. Becher and M. Neubert, Infrared Singularities of Scattering Amplitudes in Perturbative QCD, Phys. Rev. Lett. 102, 162001 (2009); Erratum, Phys. Rev. Lett. 111, 199905(E) (2013).

[4] E. Gardi and L. Magnea, Factorization constraints for soft anomalous dimensions in QCD scattering amplitudes, J. High Energy Phys. 03 (2009) 079.

[5] V. Ravindran, J. Smith, and W. L. van Neerven, Two-loop corrections to Higgs boson production, Nucl. Phys. B704, 332 (2005).

[6] S. Moch, J. A. M. Vermaseren, and A. Vogt, Three-loop results for quark and gluon form-factors, Phys. Lett. B 625 , 245 (2005).

[7] N. Rana, Perturbative QCD corrections to processes at the LHC, Ph.D. thesis, Institute of Mathematical Sciences, 2016, https://www.imsc.res.in/xmlui/handle/123456789/ 394.

[8] S. Catani, S. Dittmaier, and Z. Trocsanyi, One loop singular behavior of QCD and SUSY QCD amplitudes with massive partons, Phys. Lett. B 500, 149 (2001).

[9] A. A. Penin, Two-loop photonic corrections to massive Bhabha scattering, Nucl. Phys. B734, 185 (2006).

[10] A. Mitov and S. Moch, The singular behavior of massive QCD amplitudes, J. High Energy Phys. 05 (2007) 001.
[11] T. Becher and K. Melnikov, Two-loop QED corrections to Bhabha scattering, J. High Energy Phys. 06 (2007) 084.

[12] A. Mitov, G. F. Sterman, and I. Sung, The massive soft anomalous dimension matrix at two loops, Phys. Rev. D 79, 094015 (2009).

[13] T. Becher and M. Neubert, Infrared singularities of QCD amplitudes with massive partons, Phys. Rev. D 79, 125004 (2009); Erratum 80, 109901 (2009).

[14] T. Ahmed, J. M. Henn, and M. Steinhauser, High energy behaviour of form factors, J. High Energy Phys. 06 (2017) 125.

[15] V. Ravindran, On Sudakov and soft resummations in QCD, Nucl. Phys. B746, 58 (2006).

[16] V. Ravindran, Higher-order threshold effects to inclusive processes in QCD, Nucl. Phys. B752, 173 (2006).

[17] J. Ablinger, J. Blümlein, S. Klein, C. Schneider, and F. Wißbrock, The $O\left(\alpha_{s}^{3}\right)$ massive operator matrix elements of $O\left(n_{f}\right)$ for the structure function $F_{2}\left(x, Q^{2}\right)$ and transversity, Nucl. Phys. B844, 26 (2011).

[18] J. Ablinger, A. Behring, J. Blümlein, A. De Freitas, A. Hasselhuhn, A. von Manteuffel, M. Round, C. Schneider, and F. Wißbrock, The 3-loop non-singlet heavy flavor contributions and anomalous dimensions for the structure function $F_{2}\left(x, Q^{2}\right)$ and transversity, Nucl. Phys. B886, 733 (2014).

[19] J. Ablinger, A. Behring, J. Blümlein, A. De Freitas, A. von Manteuffel, and C. Schneider, The 3-loop pure singlet heavy 
flavor contributions to the structure function $F_{2}\left(x, Q^{2}\right)$ and the anomalous dimension, Nucl. Phys. B890, 48 (2015).

[20] J. Ablinger, J. Blümlein, A. De Freitas, A. Hasselhuhn, A. von Manteuffel, M. Round, C. Schneider, and F. Wißbrock, The transition matrix element $A_{g q}(N)$ of the variable flavor number scheme at $O\left(\alpha_{s}^{3}\right)$, Nucl. Phys. B882, 263 (2014).

[21] J. Ablinger, A. Behring, J. Blümlein, A. De Freitas, A. von Manteuffel, and C. Schneider, DESY, Report No. DESY 15-112.

[22] A. Behring, I. Bierenbaum, J. Blümlein, A. De Freitas, S. Klein, and F. Wißbrock, The logarithmic contributions to the $O\left(\alpha_{s}^{3}\right)$ asymptotic massive Wilson coefficients and operator matrix elements in deeply inelastic scattering, Eur. Phys. J. C 74, 3033 (2014).

[23] J. Ablinger, J. Blümlein, A. De Freitas, A. Hasselhuhn, C. Schneider, and F. Wißbrock, Three loop massive operator matrix elements and asymptotic Wilson coefficients with two different masses, Nucl. Phys. B921, 585 (2017).

[24] J. Blümlein, J. Ablinger, A. Behring, A. De Freitas, A. von Manteuffel, and C. Schneider, Heavy flavor Wilson coefficients in deep-inelastic scattering: Recent results, Proc. Sci., QCDEV2017 (2017) 031 [arXiv:1711.07957].

[25] W. Bernreuther, R. Bonciani, T. Gehrmann, R. Heinesch, T. Leineweber, P. Mastrolia, and E. Remiddi, Two-loop QCD corrections to the heavy quark form-factors: The vector contributions, Nucl. Phys. B706, 245 (2005).

[26] W. Bernreuther, R. Bonciani, T. Gehrmann, R. Heinesch, T. Leineweber, P. Mastrolia, and E. Remiddi, Two-loop QCD corrections to the heavy quark form-factors: Axial vector contributions, Nucl. Phys. B712, 229 (2005).

[27] W. Bernreuther, R. Bonciani, T. Gehrmann, R. Heinesch, T. Leineweber, and E. Remiddi, Two-loop QCD corrections to the heavy quark form-factors: Anomaly contributions, Nucl. Phys. B723, 91 (2005).

[28] W. Bernreuther, R. Bonciani, T. Gehrmann, R. Heinesch, P. Mastrolia, and E. Remiddi, Decays of scalar and pseudoscalar Higgs bosons into fermions: Two-loop QCD corrections to the Higgs-quark-antiquark amplitude, Phys. Rev. D 72, 096002 (2005).

[29] J. Gluza, A. Mitov, S. Moch, and T. Riemann, The QCD form factor of heavy quarks at NNLO, J. High Energy Phys. 07 (2009) 001.

[30] J. Ablinger, A. Behring, J. Blümlein, G. Falcioni, A. De Freitas, P. Marquard, N. Rana, and C. Schneider, Heavy quark form factors at two loops, Phys. Rev. D 97, 094022 (2018).

[31] J. Henn, A. V. Smirnov, V. A. Smirnov, and M. Steinhauser, Massive three-loop form factor in the planar limit, J. High Energy Phys. 01 (2017) 074.

[32] J. Ablinger, J. Blümlein, P. Marquard, N. Rana, and C. Schneider, Heavy quark form factors at three loops in the planar limit, Phys. Lett. B 782, 528 (2018).

[33] J. Ablinger, J. Blümlein, P. Marquard, N. Rana, and C. Schneider, Automated solution of first order factorizable systems of differential equations in one variable, DESY, Report No. DESY 18-053.

[34] R. N. Lee, A. V. Smirnov, V. A. Smirnov, and M. Steinhauser, Three-loop massive form factors: Complete light-fermion and large- $\mathrm{N}_{c}$ corrections for vector, axialvector, scalar and pseudo-scalar currents, J. High Energy Phys. 05 (2018) 187.
[35] J.C. Collins, Algorithm to compute corrections to the Sudakov form-factor, Phys. Rev. D 22, 1478 (1980); Sudakov form-factors, Adv. Ser. Dir. High Energy Phys. 5, 573 (1989).

[36] O. V. Tarasov, A. A. Vladimirov, and A. Y. Zharkov, The Gell-Mann-Low function of QCD in the three loop approximation, Phys. Lett. B 93, 429 (1980).

[37] S. A. Larin and J. A. M. Vermaseren, The three loop QCD beta function and anomalous dimensions, Phys. Lett. B 303, 334 (1993).

[38] T. van Ritbergen, J. A. M. Vermaseren, and S. A. Larin, The four loop beta function in quantum chromodynamics, Phys. Lett. B 400, 379 (1997).

[39] M. Czakon, The four-loop QCD $\beta$-function and anomalous dimensions, Nucl. Phys. B710, 485 (2005).

[40] P. A. Baikov, K. G. Chetyrkin, and J. H. Kühn, Five-Loop Running of the QCD Coupling Constant, Phys. Rev. Lett. 118, 082002 (2017).

[41] F. Herzog, B. Ruijl, T. Ueda, J. A. M. Vermaseren, and A. Vogt, The five-loop $\beta$-function of Yang-Mills theory with fermions, J. High Energy Phys. 02 (2017) 090.

[42] T. Luthe, A. Maier, P. Marquard, and Y. Schröder, The fiveloop $\beta$-function for a general gauge group and anomalous dimensions beyond Feynman gauge, J. High Energy Phys. 10 (2017) 166.

[43] S. Moch, J. A. M. Vermaseren, and A. Vogt, The three loop splitting functions in QCD: The nonsinglet case, Nucl. Phys. B688, 101 (2004).

[44] A. Vogt, S. Moch, and J. A. M. Vermaseren, The three-loop splitting functions in QCD: The singlet case, Nucl. Phys. B691, 129 (2004).

[45] J. M. Henn, A. V. Smirnov, V. A. Smirnov, and M. Steinhauser, A planar four-loop form factor and cusp anomalous dimension in QCD, J. High Energy Phys. 05 (2016) 066.

[46] J. Davies, A. Vogt, B. Ruijl, T. Ueda, and J. A. M. Vermaseren, Large- $n_{f}$ contributions to the four-loop splitting functions in QCD, Nucl. Phys. B915, 335 (2017).

[47] J. Henn, A. V. Smirnov, V. A. Smirnov, M. Steinhauser, and R. N. Lee, Four-loop photon quark form factor and cusp anomalous dimension in the large- $N_{c}$ limit of QCD, J. High Energy Phys. 03 (2017) 139.

[48] R. N. Lee, A. V. Smirnov, V. A. Smirnov, and M. Steinhauser, The $n_{f}^{2}$ contributions to fermionic four-loop form factors, Phys. Rev. D 96, 014008 (2017).

[49] S. Moch, B. Ruijl, T. Ueda, J. A. M. Vermaseren, and A. Vogt, Four-loop non-singlet splitting functions in the planar limit and beyond, J. High Energy Phys. 10 (2017) 041.

[50] S. Moch, B. Ruijl, T. Ueda, J. A. M. Vermaseren, and A. Vogt, On quartic colour factors in splitting functions and the gluon cusp anomalous dimension, Phys. Lett. B 782, 627 (2018).

[51] D. J. Broadhurst, N. Gray, and K. Schilcher, Gauge invariant on-shell Z(2) in QED, QCD and the effective field theory of a static quark, Z. Phys. C 52, 111 (1991).

[52] J. A. M. Vermaseren, S. A. Larin, and T. van Ritbergen, The 4-loop quark mass anomalous dimension and the invariant quark mass, Phys. Lett. B 405, 327 (1997).

[53] K. Melnikov and T. van Ritbergen, The three loop on-shell renormalization of QCD and QED, Nucl. Phys. B591, 515 (2000). 
[54] J. A. Gracey Three loop $\overline{\mathrm{MS}}$ tensor current anomalous dimension in QCD, Phys. Lett. B 488, 175 (2000).

[55] P. Marquard, L. Mihaila, J. H. Piclum, and M. Steinhauser, Relation between the pole and the minimally subtracted mass in dimensional regularization and dimensional reduction to three-loop order, Nucl. Phys. B773, 1 (2007).

[56] T. Luthe, A. Maier, P. Marquard, and Y. Schröder, Five-loop quark mass and field anomalous dimensions for a general gauge group, J. High Energy Phys. 01 (2017) 081.

[57] P. A. Baikov, K. G. Chetyrkin, and J. H. Kühn, Five-loop fermion anomalous dimension for a general gauge group from four-loop massless propagators, J. High Energy Phys. 04 (2017) 119.

[58] P. Marquard, A. V. Smirnov, V. A. Smirnov, and M. Steinhauser, Quark Mass Relations to Four-Loop Order in Perturbative QCD, Phys. Rev. Lett. 114, 142002 (2015).

[59] P. Marquard, A. V. Smirnov, V. A. Smirnov, M. Steinhauser, and D. Wellmann, $\overline{\mathrm{MS}}$-on-shell quark mass relation up to four loops in QCD and a general $\mathrm{SU}(N)$ gauge group, Phys. Rev. D 94, 074025 (2016).

[60] P. Marquard, A. V. Smirnov, V. A. Smirnov, and M. Steinhauser, Four-loop wave function renormalization in QCD and QED, Phys. Rev. D 97, 054032 (2018).
[61] M. Neubert, Renormalization-group improved calculation of the $B \rightarrow X(s) \gamma$ branching ratio, Eur. Phys. J. C 40, 165 (2005).

[62] E. Gardi, Radiative and semileptonic B meson decay spectra: Sudakov resummation beyond logarithmic accuracy and the pole mass, J. High Energy Phys. 04 (2004) 049, and unpublished work.

[63] A. Grozin, J. M. Henn, G. P. Korchemsky, and P. Marquard, Three Loop Cusp Anomalous Dimension in QCD, Phys. Rev. Lett. 114, 062006 (2015).

[64] T. Ahmed, M. Mahakhud, N. Rana, and V. Ravindran, Drell-Yan Production at Threshold to Third Order in QCD, Phys. Rev. Lett. 113, 112002 (2014).

[65] T. Ahmed, N. Rana, and V. Ravindran, Higgs boson production through $b \bar{b}$ annihilation at threshold in $\mathrm{N}^{3} \mathrm{LO}$ QCD, J. High Energy Phys. 10 (2014) 139.

[66] A. Devoto and D. W. Duke, Table of integrals and formulae for Feynman diagram calculations, Riv. Nuovo Cimento 7N6, 1 (1984).

[67] L. Lewin, Dilogarithms and Associated Functions (Macdonald, London, 1958).

[68] L. Lewin, Polylogarithms and Associated Functions (North Holland, New York, 1981). 